\title{
The Dynamical Degrees of Freedom in Spatially Homogeneous Cosmology ${ }^{\star}$
}

\author{
Robert T. Jantzen \\ Department of Physics, University of California, Berkeley, California 94720, USA
}

\begin{abstract}
The true analogues of superspace and conformal superspace for spatially homogeneous cosmology are introduced and discussed in relation to the kinematics of the evolution of Cauchy data from a spatially homogeneous initial value surface using a spatially homogeneous lapse function. Having fixed the slicing of spatially homogeneous spacetimes to be the natural one, an obvious restriction on the freedom of choice of the shift vector field occurs, and its relation to the three-dimensional diffeomorphism gauge group of the problem is explained. In this context the minimal distortion shift equation of Smarr and York naturally arises. Finally these ideas are used to simplify the dynamics.
\end{abstract}

\section{Preliminaries}

We first introduce a good deal of notation and standard results concerning a Lie group and its associated Lie algebras and groups ${ }^{1}$. Let $G$ be a simply connected $n$ dimensional Lie group ( $n=3$ will be our principal application) with identity element $u_{0}, \mathscr{D}(G)$ its diffeomorphism group, $\mathfrak{X}(G)$ the Lie algebra of vector fields on $G$ and $\mathfrak{g}$ and $\tilde{\mathfrak{g}}$ the respectively left invariant and right invariant $n$-dimensional Lie subalgebras of $\mathfrak{X}(G)$.

Let $\mathfrak{X}(G)^{*}$ be the space of 1 -forms on $G$ and $\mathfrak{g}^{*}$ and $\tilde{\mathfrak{g}}^{*}$ the respectively left invariant and right invariant $n$-dimensional subspaces of $\mathfrak{X}(G)^{*}$. These may be identified with the dual spaces of $\mathfrak{g}$ and $\tilde{\mathfrak{g}}$, and the entire space of left or right invariant tensor fields on $G$ may be identified with the tensor algebra over $\mathfrak{g}$ or $\tilde{\mathfrak{g}}$ respectively. $\mathfrak{g}$ is usually called the Lie algebra of $G$. A choice of basis $e=\left\{e_{a}\right\}$ of $\mathfrak{g}$ determines a basis $\tilde{e}=\left\{\tilde{e}_{a}\right\}$ of $\tilde{\mathfrak{g}}$ (uniquely defined so that $e$ and $\tilde{e}$ coincide at the identity) and dual bases $\left\{\omega^{a}\right\}$ and $\left\{\tilde{\omega}^{a}\right\}$ of the corresponding dual spaces. Both $e$ and $\tilde{e}$ are global frames on $G$ with respective dual frames $\left\{\omega^{a}\right\}$ and $\left\{\tilde{\omega}^{a}\right\}$.

The group of automorphisms of $G$

$$
\operatorname{Aut}(G)=\left\{h \in \mathscr{D}(G) \mid h\left(u_{1} u_{2}\right)=h\left(u_{1}\right) h\left(u_{2}\right) ; \forall u_{1}, u_{2} \in G\right\}
$$

* Work supported by NSF grant MCS-7621525 
is a Lie group acting on $G$; let $\operatorname{aut}(G) \subset \mathfrak{X}(G)$ be the Lie algebra of generators of this action. The subgroup of inner automorphisms of $G$ or the adjoint group is

$$
A D(G)=\left\{A D_{u} \mid u \in G\right\}
$$

where $A D_{u}=L_{u^{\circ}} \circ R_{u^{-1}}=R_{u^{-1}} \circ L_{u}$ and $L_{u_{1}}\left(u_{2}\right)=u_{1} u_{2}=R_{u_{2}}\left(u_{1}\right)$ are left and right translation respectively.

Let $G L(\mathfrak{g}) \subset \mathfrak{g l}(\mathfrak{g})$ be the group of vector space automorphisms of $\mathfrak{g}$ and $\mathfrak{g l}(\mathfrak{g})$ the Lie algebra of linear transformations of $\mathfrak{g}$ into itself. The group of Lie algebra automorphisms of $\mathfrak{g}$

$$
\operatorname{Aut}(\mathfrak{g})=\{A \in \mathrm{GL}(\mathfrak{g}) \mid A[X, Y]=[A X, A Y] ; \forall X, Y \in \mathfrak{g}\}
$$

has as its Lie algebra the derivations of $\mathfrak{g}$

$$
\operatorname{aut}(\mathfrak{g})=\operatorname{Der}(\mathfrak{g})=\{B \in \mathfrak{g l}(\mathfrak{g}) \mid B[X, Y]=[B X, Y]+[X, B Y] ; \forall X, Y \in \mathfrak{g}\} .
$$

The group of inner automorphisms of $\mathfrak{g}$ or linear adjoint group is designated by $\operatorname{Ad}(G)$ and is the connected Lie subgroup of Aut( $\mathfrak{g})$ obtained by exponentiating the Lie algebra $\operatorname{ad}(\mathfrak{g})$ of inner derivations of $\mathfrak{g}$ (the adjoint Lie algebra):

$$
\operatorname{ad}(\mathfrak{g})=\{\operatorname{ad}(X) \mid X \in \mathfrak{g}\} \subset \operatorname{aut}(\mathfrak{g}),
$$

where $\operatorname{ad}(X) Y=[X, Y]$ for $X, Y \in \mathfrak{g}$. ad: $\mathfrak{g} \rightarrow \mathfrak{g l}(\mathfrak{g})$ is a homomorphism called the adjoint representation of $\mathfrak{g}$. We will also use the notation $\operatorname{ad}(X) Y=[X, Y]$ for the adjoint representation of $\mathfrak{X}(G)$. (In each case the bracket is the Lie bracket of vector fields.) If $H$ is a subgroup of $\mathrm{GL}(\mathfrak{g})$ with Lie algebra $\mathfrak{h} \subset \mathfrak{g l}(\mathfrak{g})$, let $S H$ denote the unimodular subgroup of $H$ consisting of the linear transformations of $H$ with unit determinant and let $\mathfrak{s h}$ be the corresponding Lie subalgebra of $\mathfrak{h}$ consisting of its traceless elements. For example, SAut(g) is the special automorphism group of $\mathfrak{g}$ and $\mathfrak{s a u t}(\mathfrak{g})$ is its Lie algebra.

A choice of basis $e=\left\{e_{a}\right\}$ of $\mathfrak{g}$ determines an isomorphism $e: \mathfrak{g} \rightarrow R^{n}$ and a corresponding isomorphism from $\mathfrak{g l}(\mathfrak{g}) \rightarrow \mathfrak{g l}(n, R)$ and from $\mathrm{GL}(\mathfrak{g}) \rightarrow \mathrm{GL}(n, R)$. Denote by $\operatorname{Aut}_{e}(\mathfrak{g})$, aut $(\mathfrak{g})$, etc., the images of the various subgroups of $G L(\mathfrak{g})$ in $\operatorname{GL}(n, R)$ and Lie subalgebras of $\mathfrak{g l}(\mathfrak{g})$ in $\operatorname{gl}(n, R)$, and if $u \in G, X \in \mathfrak{g}$ and $\xi \in$ aut $(G)$, let $\operatorname{Ad}_{e}(u) \in \operatorname{Ad}_{e}(G), \operatorname{ad}_{e}(X) \in \operatorname{ad}_{e}(\mathfrak{g})$ and $\operatorname{ad}_{e}(\xi) \in \mathfrak{a u t}_{e}(\mathfrak{g})^{1}$ be the corresponding matrices with respect to the basis $e$. If $C_{b c}^{a}=\omega^{a}\left(\left[e_{b}, e_{c}\right]\right)$ are the components of the structure constant tensor of $\mathfrak{g}$ in this basis, then define $\mathbf{k}_{a}=\left(C^{b}{ }_{a c}\right)=\operatorname{ad}_{e}\left(e_{a}\right) \in \mathfrak{g l}(n, R)$. Recall that $e$ is a global frame on $G$ with dual frame $\left\{\omega^{a}\right\}$; the same is true of $\tilde{e}$ and $\left\{\tilde{\omega}^{a}\right\}$. The two frames are related by the matrix of the adjoint representation of $G$ with respect to the basis $e:^{1}$

$$
\begin{aligned}
& \mathbf{R}(u)=\operatorname{Ad}_{e}(u), \quad u \in G \\
& \tilde{e}_{a}=R^{-1 b}{ }_{a} e_{b} \quad \tilde{\omega}^{a}=R^{a}{ }_{b} \omega^{b} \\
& \mathbf{R}^{-1} d \mathbf{R}=\mathbf{k}_{a} \omega^{a} \quad d \mathbf{R} \mathbf{R}^{-1}=\mathbf{k}_{a} \tilde{\omega}^{a} \\
& {\left[e_{a}, e_{b}\right]=C_{a b}^{c} e_{c} \quad\left[e_{a}, \tilde{e}_{b}\right]=0 \quad\left[\tilde{e}_{a}, \tilde{e}_{b}\right]=-C_{a b}^{c} \tilde{e}_{c} .}
\end{aligned}
$$

1 See the next paragraph in the text 
For the convenience of the reader, we note that the Lie algebra automorphism condition in the form

$$
[X, Y]=A^{-1}[A X, A Y] \quad \forall X, Y \in \mathfrak{g} ; A \in \operatorname{Aut}(\mathfrak{g})
$$

has the following expression in terms of the basis $e$ :

$$
C_{b c}^{a}=A^{-1 a}{ }_{d} C_{f g}^{d} A_{b}^{f} A^{g}{ }_{c} \quad A_{b}^{a}=\omega^{a}\left(A e_{b}\right) .
$$

Aut $_{e}(\mathfrak{g})$ is the set of all $\mathbf{A} \in \mathrm{GL}(n, R)$ satisfying this condition.

If $h \in \operatorname{Aut}(G)$, the vector field $h X$ obtained from $X \in \mathfrak{g}$ by dragging along by $h$ also lies in $\mathfrak{g}$, so Aut $(\mathfrak{g})$ acts naturally on $\mathfrak{g}$ as a subgroup of $\mathrm{GL}(\mathfrak{g})$ [since dragging along is a representation of $\mathscr{D}(G)]$. In fact this is an isomorphism from $\operatorname{Aut}(G)$ onto Aut $(\mathfrak{g})$ and from $A D(G)$ onto $\operatorname{Ad}(G)$. If $\operatorname{Ad}(u)$ is the image of the inner automorphism $A D_{u}$ under this isomorphism, then $\mathrm{Ad}: G \rightarrow \mathrm{GL}(\mathrm{g})$ is a homomorphism called the adjoint representation of $G$. Similarly the restriction of the adjoint representation of $\mathfrak{X}(G)$ to $\mathfrak{a u t}(G)$ when acting on $\mathfrak{g}$ viewed as a Lie subalgebra of $\mathfrak{X}(G)$ is an isomorphism onto aut $(\mathfrak{g})$. In other words for each $\xi \in \mathfrak{a u t}(G)$, $\operatorname{ad}(\xi)$ acts as a derivation on $\mathfrak{g}$ and every derivation of $\mathfrak{g}$ may be obtained in this way.

Left and right translations also act on $\mathfrak{g}$ by dragging along. By definition left translation acts as the identity on $\mathfrak{g}$, while for the same reason $R_{u^{-1}}$ and $A D_{u}$ $=R_{u^{-1}} L_{u}$ have the same action on $\mathfrak{g}$, namely they induce the linear transformation $\operatorname{Ad}(u)$ on $\mathfrak{g}$. For $X \in \mathfrak{g}$ there is a unique $\tilde{X} \in \tilde{\mathfrak{g}}$ such that $X\left(u_{0}\right)=\tilde{X}\left(u_{0}\right)$. The statement that $\mathfrak{g}$ and $\tilde{\mathfrak{g}}$ generate the right and left translations respectively means that

$$
R_{\exp t X}=X_{t}, \quad L_{\exp t X}=\tilde{X}_{t},
$$

where $\left\{X_{t} \mid t \in R\right\}$ is the one-parameter group of diffeomorphisms generated by the vector field $X$ (the flow of $X$ ) and the exponential map exp : $\mathfrak{g} \rightarrow G$ may be defined by $\exp X=X_{1}\left(u_{0}\right)$. In particular, the action of $X_{t}$ on $\mathfrak{g}$ by dragging along is equivalent to the action of $\operatorname{Ad}(\exp -t X)=\exp (-t \operatorname{tad}(X))$ on $g$. In fact if $X(t)$ is a parametrized curve in $\mathfrak{g}$, i.e. a "time-dependent" left invariant vector field on $G$, then it generates a curve $h(t)$ in $\mathscr{D}(G)$ called its flow [2] that will be a curve of right translations which when acting on $\mathfrak{g}$ by dragging along will be equivalent to the action of a curve in the linear adjoint group. Similarly if $X(t)$ is a parametrized curve in aut $(G)$, its flow will be a curve of automorphisms of $G$ which when acting on $\mathfrak{g}$ by dragging along will be equivalent to the action of a curve in Aut $(\mathfrak{g})$. Since Aut(g) is a subgroup of $\mathrm{GL}(\mathfrak{g})$, it acts naturally on the tensor algebra over $\mathfrak{g}$ (left invariant tensor fields on $G$ ). It is just this action which is produced by dragging along by $\operatorname{Aut}(G)$. Thus given a curve $X(t)$ in aut $(G)$, its flow will be a timedependent automorphism of $G$ which induces the action of the corresponding time-dependent automorphism of $\mathfrak{g}$ when it acts on the tensor algebra over $\mathfrak{g}$ by dragging along.

Let $\mathscr{M}(G)$ be the space of left invariant Riemannian metrics on $G$, naturally identifiable with the space $\mathscr{M}(\mathrm{g})$ of (positive definite) inner products on $\mathrm{g}$. Let $C$ be the abelian group $R^{+}$acting on $\mathscr{M}(\mathrm{G})$ by conformal scaling

$$
g \in \mathscr{M}(G) \mapsto c^{2} g \in \mathscr{M}(G), \quad c \in R^{+}
$$


and let $\overline{\mathscr{M}}(G)=\mathscr{M}(G) / C$ be the space of orbits of this action. Similarly let $\mathscr{M} \subset \mathrm{GL}(n, R)^{+}$be the submanifold of matrices of components in the natural basis of inner products on $R^{n}$ and $\overline{\mathscr{M}}$ its unimodular submanifold. Note that there is a natural projection map

$$
\mathbf{g} \in \mathscr{M} \mapsto \overline{\mathbf{g}}=g^{-1 / n} \mathbf{g} \in \overline{\mathscr{M}},
$$

where $g=\operatorname{det} \mathbf{g}$. A choice of basis $e$ of $\mathfrak{g}$ provides a natural identification of $\mathscr{M}(G)$ with. $\mathscr{U}$ and of $\overline{\mathscr{U}}(G)$ with $\overline{\mathscr{U}}$. It is this reason that motivated Misner to call $\mathscr{M}$ minisuperspace in the case $n=3$ [3]. DeWitt has studied $\mathscr{M}$ for $n=3$ as a certain pseudo-Riemannian manifold closely related to the dynamics of general relatively [4]. In fact $\mathscr{M}$ is just the configuration space for the dynamics of spatially homogeneous cosmology.

Aut $(G)$ acts naturally on $\mathscr{M}(G)$ by dragging along, as do the right translations; left translations have trivial action on $\mathscr{M}(G)$, i.e. are isometries of every element of $\mathscr{M}(G)$, with $\tilde{\mathfrak{g}}$ the corresponding Killing Lie algebra. The action of $\operatorname{Aut}(G)$ on $\mathscr{M}(G)$ induces the natural left action of Aut $(\mathfrak{g})$ on $\mathscr{M}(\mathfrak{g})$ and on $\overline{\mathscr{M}}(\mathfrak{g})$, while the dragging action of right translation or inner automorphism induces the natural left action of the linear adjoint group on these spaces. A choice of basis $e$ of $\mathfrak{g}$ induces the following action of $\operatorname{Aut}_{e}(\mathfrak{g})$ on $\mathscr{M}$ and $\overline{\mathscr{M}}$ :

$$
\begin{aligned}
& \mathbf{g} \in \mathscr{M} \mapsto f_{\mathbf{A}}(\mathbf{g})=\mathbf{A}^{-1 T} \mathbf{g} \mathbf{A}^{-1} \quad \mathbf{A} \in \operatorname{Aut}_{e}(\mathfrak{g}) \\
& \overline{\mathbf{g}} \in \overline{\mathscr{M}} \mapsto \bar{f}_{\mathbf{A}}(\overline{\mathbf{g}})=(\operatorname{det} \mathbf{A})^{2 / n} f_{\mathbf{A}}(\overline{\mathbf{g}}) .
\end{aligned}
$$

It is a result of simple considerations of Lie group theory that if $h \in \mathscr{D}(G)$ maps $\mathscr{M}(G)$ into itself under dragging along, then $h=L_{h\left(u_{0}\right)} h_{1}$ where $h_{1} \in \operatorname{Aut}(G)$; in other words modulo left translations, the only elements of $\mathscr{D}(G)$ which act naturally on $\mathscr{M}(G)$ are the automorphisms of $G$. The isometry classes of $\mathscr{M}(G)$ are therefore the orbits of $\operatorname{Aut}(G)$, i.e. $\mathscr{M}_{\text {true }}(G)=\mathscr{M}(G) / \operatorname{Aut}(G)$ is the space of left invariant " $n$-geometries" or "true minisuperspace", the real analogue of superspace. Similarly the "conformal isometry classes" of $\mathscr{M}(G)$ are the orbits of the action of $C \times \operatorname{Aut}(G)$ or equivalently the orbits of $\overline{\mathscr{M}}(G)$ under the action of $\operatorname{Aut}(G)$, i.e. "true conformal minisuperspace" is $\overline{\mathscr{M}}_{\text {true }}(G)=\overline{\mathscr{M}}(G) / \operatorname{Aut}(G)$ $\sim \mathscr{M}(G) /(C \times \operatorname{Aut}(G))$. In accordance with the work of York we call $\overline{\mathscr{M}}_{\text {true }}(G)$ the space of dynamical degrees of freedom [5] and its dimension the number of dynamical degrees of freedom possessed by left invariant Riemannian metrics on $G$.

True minisuperspace has the same property as superspace [6], namely that it is not a manifold but a stratification of manifolds, since the orbits may have varying dimension. "Generic minisuperspace" is the submanifold of $\mathscr{M}(G)$ on which the orbit dimension is maximum and projects onto the generic submanifold of true minisuperspace. The isotropy group $I_{g}$ at $g \in \mathscr{M}(G)$ of the action of Aut $(G)$ consists of those automorphisms of $G$ which are isometries of $g$ and the elements of the Lie subalgebra of aut $(G)$ corresponding to the Lie algebra of $I_{g}$ are Killing vector fields of $g$. Therefore the remainder of minisuperspace necessarily has higher symmetry because of the increase in the dimension of the isotropy group, unless generic minisuperspace already has maximum symmetry. Let $\mathscr{I}_{g}$ be the isotropy group at $g \in \operatorname{Riem}(G)$ of the action of $\mathscr{D}(G)$ on the space of Riemannian metrics on $G$. If $g \in \mathscr{M}(G), \mathscr{I}_{g}$ not only contains the right translations $L(G)$ and the isotropy 
group $I_{g}$ which may or may not be trivial, but may also contain additional diffeomorphisms which do not mapg or $\mathscr{M}(G)$ into themselves, although there may be submanifolds of $\mathscr{M}(G)$ on which they do act. The corresponding Killing vector fields will not lie in $\operatorname{aut}(G)$ and will vary from point to point in $\mathscr{M}(G)$.

\section{Three-dimensional Lie Groups}

We now specialize to $n=3$ for which the components of the structure constant tensor of $\mathfrak{g}$ decompose in the following well known way [7]:

$$
\begin{aligned}
C_{b c}^{a} & =\varepsilon_{b c d} n^{a d}+a_{f} \delta_{b c}^{f a}, \\
n^{a b} & =\frac{1}{2} C^{(a}{ }_{c d} \varepsilon^{b) c d}, \quad a_{b}=\frac{1}{2} C_{b a}^{a}, \quad a_{b} n^{b a}=0=a_{c} C_{a b}^{c} .
\end{aligned}
$$

$n^{a b}$ are the components of a symmetric second rank tensor density of weight one over $\mathfrak{g}$, while $a_{b}$ are the components of a covector over $\mathfrak{g}$, i.e. an element of $\mathfrak{g}^{*}$. We will refer to these components as in "standard diagonal form" if the matrix of components $n^{a b}$ is diagonal $\left(\mathbf{n}=\operatorname{diag}\left(n^{(1)}, n^{(2)}, n^{(3)}\right)\right)$ and $a_{b}=a \delta^{3}{ }_{b}$ with $a \geqq 0$, in which case the contracted Jacobi identity reduces to $a n^{(3)}=0$.

Let $\mathscr{C}$ be the six-dimensional submanifold of $R^{3} \times R^{3} \times R^{3}$ consisting of components $C_{b c}^{a}=-C^{a}{ }_{c b}$ satisfying the Jacobi identity $C^{e}{ }_{d[a} C^{d}{ }_{b c]}=0$. GL $(3, R)$ acts naturally on this space on the left:

$$
C_{b c}^{a} \mapsto A_{d}^{a} C_{f g}^{d} A^{-1 f}{ }_{b} A^{-1 g}{ }_{c}, \quad \mathbf{A} \in \mathrm{GL}(3, R),
$$

or in terms of $n^{a b}$ and $a_{b}$ :

$$
n^{a b} \mapsto(\operatorname{det} \mathbf{A})^{-1} A_{c}^{a} n^{c d} A_{d}^{b}, \quad a_{b} \mapsto a_{c} A^{-1 c}{ }_{b} .
$$

When $a_{b} \neq 0$, a scalar $h$ is defined by the equation:

$$
a_{a} a_{b}=\frac{1}{2} h \varepsilon_{a c d} \varepsilon_{b f g} n^{c f} n^{d g},
$$

which reduces to $a^{2}=h n^{(1)} n^{(2)}$ when $C_{b c}^{a}$ is in standard diagonal form.

By definition, the isotropy group $I_{C}$ at a point $C \in \mathscr{C}$ is just the matrix automorphism group $\operatorname{Aut}_{e}(\mathfrak{g})$ of a three-dimensional Lie algebra with respect to a basis $e=\left\{e_{a}\right\}$ in which $C_{b c}^{a}$ are the components of the structure constant tensor of $\mathfrak{g}$. Secondly, the orbits of the action of $\operatorname{GL}(3, R)$ on $\mathscr{C}$ are just the equivalence classes of structure constant tensors under the isomorphism relation. The description of the orbit space has been called the Bianchi-Behr classification of threedimensional Lie algebras [7]. The orbits are characterized by the rank and absolute value of the signature of $\mathbf{n}$, the vanishing or nonvanishing of $a_{b}$ and the invariant $h$ when defined. It is useful to select a canonical representative for each orbit or "Bianchi type". This is easily done by first reducing the structure constant tensor to standard diagonal form, normalizing $a$ (if nonzero) to unity when $h$ is undefined and by further reducing $\mathbf{n}$ to its Sylvester form (except for Type II where a permuted form proves more convenient). We will refer to the canonical representative for each type as "canonical components" and call a "canonical basis" any basis $e$ of a Lie algebra $g$ with respect to which its structure constant tensor has canonical components and the various matrix Lie algebras and groups associated with $\mathfrak{g}$ in the basis $e$ the canonical such Lie algebra or group. Table 1 
lists the canonical components for each Bianchi type together with the dimensions of the canonical adjoint group $\operatorname{Ad}_{e}(G)$ and canonical automorphism group Aut $_{e}(\mathrm{~g})$ and finally the dimension of the orbit to which each belongs, which is just 9$\operatorname{dim}\left(\operatorname{Aut}_{e}(\mathfrak{g})\right)$. The Roman numerals were assigned by Bianchi [8] who used a different classification scheme than the present one which is due to Behr [7].

Table 1

\begin{tabular}{|c|c|c|c|c|c|c|c|c|c|}
\hline & & $n^{(1)}$ & $n^{(2)}$ & $n^{(3)}$ & $a$ & $h$ & $\operatorname{dim}\left(\operatorname{Ad}_{c^{\prime}}(G)\right)$ & $\operatorname{dim}\left(\operatorname{Aut}_{e}(g)\right)$ & dim(orbit) \\
\hline \multirow[t]{6}{*}{ Class A } & I & () & () & 0 & 0 & $\ldots$ & 0 & 9 & 0 \\
\hline & II & 0 & 0 & 1 & 0 & $\ldots$ & 2 & 6 & 3 \\
\hline & $\mathrm{VI} \mathrm{I}_{0}$ & 1 & -1 & 0 & () & 0 & 3 & 4 & 5 \\
\hline & $\mathrm{VII}_{0}$ & 1 & 1 & 0 & () & 0 & 3 & 4 & 5 \\
\hline & VIII & 1 & 1 & -1 & 0 & 0 & 3 & 3 & 6 \\
\hline & IX & 1 & 1 & 1 & 0 & 0 & 3 & 3 & 6 \\
\hline \multirow[t]{2}{*}{ Class B } & V & 0 & 0 & 0 & 1 & - & 3 & 6 & 3 \\
\hline & IV & 1 & 0 & 0 & 1 & $\ldots$ & 3 & 4 & 5 \\
\hline \multicolumn{2}{|c|}{$H I=V I-1$} & 1 & -1 & 0 & 1 & -1 & 2 & 4 & 5 \\
\hline \multicolumn{2}{|c|}{$V I_{h \neq 0}-1$} & 1 & -1 & 0 & $a$ & $-a^{2}$ & 3 & 4 & 5 \\
\hline \multicolumn{2}{|c|}{$\mathrm{V} \mathrm{I}_{h \mp 0}$} & 1 & 1 & 0 & $a$ & $a^{2}$ & 3 & 4 & 5 \\
\hline
\end{tabular}

The types for which $a_{b}=0$ are called class $\mathrm{A}$ and the remaining are called Class B. For later purposes it is also useful to call Types I, II, III, V, and VI $-1 / 9$ degenerate and the remaining types nondegenerate.

It is convenient to introduce the notation $\mathbf{e}^{b}{ }_{a}$ for the matrix whose only nonvanishing component is a one in the $a^{\text {th }}$ row and $b^{\text {th }}$ column, so that a matrix with components $A^{a}{ }_{b}$ may be written $\mathbf{A}=A^{a}{ }_{b} \mathbf{e}_{a}^{b} .\left\{\mathbf{e}^{b}{ }_{a}\right\}$ is the natural basis of gl $(3, R)$. For all but Types I, II, and III, the adjoint representation ad is a Lie algebra isomorphism so one can imbed in $\operatorname{GL}(3, R)^{+}$a Lie group of each Bianchi type [namely the canonical adjoint $\left.\operatorname{group}_{\operatorname{Ad}_{e}}(G)\right]$ by exponentiating the canonical adjoint Lie algebra $\operatorname{ad}_{e}(\mathfrak{g})$. This has the canonical basis $\left\{\mathbf{k}_{a}\right\}$ where we recall that $\mathbf{k}_{a}=C^{b}{ }_{a c} \mathbf{e}_{b}^{c}$ and $\operatorname{Tr} \mathbf{k}_{a}=2 a_{a}$. For a structure constant tensor in standard diagonal form these are explicitly:

$$
\begin{aligned}
\mathbf{k}_{1} & =-n^{(2)} \mathbf{e}^{3}{ }_{2}+n^{(3)} \mathbf{e}^{2}{ }_{3}-a \mathbf{e}^{3}{ }_{1} \\
\mathbf{k}_{2} & =-n^{(3)} \mathbf{e}^{1}{ }_{3}+n^{(1)} \mathbf{e}^{3}{ }_{1}-a \mathbf{e}^{3}{ }_{2} \\
\mathbf{k}_{3} & =-n^{(1)} \mathbf{e}^{2}{ }_{1}+n^{(2)} \mathbf{e}^{1}{ }_{2}+a\left(\mathbf{e}^{1}{ }_{1}+\mathbf{e}^{2}{ }_{2}\right) \\
{\left[\mathbf{k}_{2}, \mathbf{k}_{3}\right] } & =n^{(1)} \mathbf{k}_{1}-a \mathbf{k}_{2} \\
{\left[\mathbf{k}_{3}, \mathbf{k}_{1}\right] } & =n^{(2)} \mathbf{k}_{2}+a \mathbf{k}_{1} \\
{\left[\mathbf{k}_{1}, \mathbf{k}_{2}\right] } & =n^{(3)} \mathbf{k}_{3} .
\end{aligned}
$$

The matrix $\mathbf{k}_{3}$ is responsible for the nonunimodularity of the Class B adjoint group. If we let $\mathbf{I}^{(a)}=\mathbf{1}-\mathbf{e}^{a}{ }_{a}$ and let $\mathbf{k}_{a}^{0}$ be the matrix obtained from $\mathbf{k}_{a}$ by setting the structure component $a$ to zero, then $\mathbf{k}_{3}=\mathbf{k}_{3}^{0}+a \mathbf{I}^{(3)}$ isolates the only nonzero diagonal components of any of the $\mathbf{k}_{a}$. 
Canonical coordinates of the second kind [1] lead to the following parametrization of the adjoint matrix $\mathbf{R}$; the exponentiation can be done explicitly:

$$
\begin{aligned}
\mathbf{R}(x) & =e^{x^{1} \mathbf{k}_{1}} e^{x^{2} \mathbf{k}_{2}} e^{x^{3} \mathbf{k}_{3}} \\
& =\left[\begin{array}{ccc}
1 & 0 & -a x^{1} \\
0 & c_{1} & -n^{(2)} s_{1} \\
0 & n^{(3)} s_{1} & c_{1}
\end{array}\right]\left[\begin{array}{ccc}
c_{2} & 0 & n^{(1)} s_{2} \\
0 & 1 & -a x^{2} \\
-n^{(3)} s_{2} & 0 & c_{2}
\end{array}\right]\left[\begin{array}{ccc}
c_{3} e^{a x^{3}} & -n^{(1)} s_{3} e^{a x^{3}} & 0 \\
n^{(2)} s_{3} e^{a x^{3}} & c_{3} e^{a x^{3}} & 0 \\
0 & 0 & 1
\end{array}\right]
\end{aligned}
$$

Note that $\operatorname{det} \mathbf{R}=e^{2 a x^{3}}$.

The following abbreviations and identities are used; $(a, b, c)$ is any cyclic permutation of $(1,2,3)$ :

$$
\begin{aligned}
m^{(a)} & =\left(-n^{(b)} n^{(c)}\right)^{1 / 2} \quad \tilde{m}^{(a)}=\left(n^{(b)} n^{(c)}\right)^{1 / 2} \\
c_{a} & =\cosh m^{(a)} x^{a}=\cos \tilde{m}^{(a)} x^{a} \\
S_{a} & =\left(m^{(a)}\right)^{-1} \sinh m^{(a)} x^{a}=\left(\tilde{m}^{(a)}\right)^{-1} \sin \tilde{m}^{(a)} x^{a} \\
\lim _{m^{(a)} \rightarrow 0}\left(c_{a}, s_{a}\right) & =\left(1, x^{a}\right) \\
\left(c_{a}\right)^{2}-\left(m^{(a)} s_{a}\right)^{2} & =\left(c_{a}\right)^{2}+\left(\tilde{m}^{(a)} s_{a}\right)^{2}=1 \\
d c_{a} & =\left(m^{(a)}\right)^{2} s_{a} d x^{a} \quad d s_{a}=c_{a} d x^{a} .
\end{aligned}
$$

The left and right invariant dual frames on $\operatorname{Ad}_{e}(G)$ determined by the basis $\left\{\mathbf{k}_{a}\right\}$ of its Lie algebra $\mathrm{ad}_{e}(\mathrm{~g})$ are computable from the relations:

$$
\mathbf{R}^{-1} d \mathbf{R}=\mathbf{k}_{a} \omega^{a} \quad d \mathbf{R} \mathbf{R}^{-1}=\mathbf{k}_{a} \tilde{\omega}^{a} .
$$

The invariant frames themselves may be constructed using the duality relations. The result is :

$$
\begin{array}{ll}
\omega^{1}=e^{-a x^{3}}\left(c_{2} c_{3} d x^{1}+n^{(1)} s_{3} d x^{2}\right) & e_{1}=e^{a x^{3}}\left(n^{(2)} s_{3} \partial_{2}+c_{3} c_{2}{ }^{-1}\left(\partial_{1}-n^{(3)} s_{2} \partial_{3}\right)\right) \\
\omega^{2}=e^{-a x^{3}}\left(-n^{(2)} c_{2} s_{3} d x^{1}+c_{3} d x^{2}\right) & e_{2}=e^{a x^{3}}\left(c_{3} \partial_{2}-n^{(1)} s_{3} c_{2}{ }^{-1}\left(\partial_{1}-n^{(3)} s_{2} \partial_{3}\right)\right) \\
\omega^{3}=n^{(3)} s_{2} d x^{1}+d x^{3} & e_{3}=\partial_{3} \\
\tilde{\omega}^{1}=d x^{1}+\left(n^{(1)} s_{2}-a x^{1}\right) d x^{3} & \tilde{e}_{1}=\partial_{1} \\
\tilde{\omega}^{2}=c_{1} d x^{2}-\left(n^{(2)} s_{1} c_{2}+a x^{2}\right) d x^{3} & \tilde{e}_{2}=c_{1} \partial_{2}-n^{(3)} s_{1} c_{2}{ }^{-1}\left(\partial_{3}-n^{(1)} s_{2} \partial_{1}\right) \\
\tilde{\omega}^{3}=n^{(3)} s_{1} d x^{2}+c_{1} c_{2} d x^{3} & \tilde{e}_{3}=n^{(2)} s_{1} \partial_{2}+c_{1} c_{2}{ }^{-1}\left(\partial_{3}-n^{(1)} s_{2} \partial_{1}\right) \\
& \quad+a\left(x^{1} \partial_{1}+x^{2} \partial_{2}\right) \\
\omega^{1} \wedge \omega^{2} \wedge \omega^{3}=e^{-2 a x^{3}} c_{2} d x^{1} \wedge d x^{2} \wedge d x^{3} & \\
\tilde{\omega}^{1} \wedge \tilde{\omega}^{2} \wedge \tilde{\omega}^{3}=c_{2} d x^{1} \wedge d x^{2} \wedge d x^{3} . &
\end{array}
$$

Similar formulas may also be obtained for other types of exponential coordinates. Although this procedure is not valid for Types I, II and III since $\mathbf{k}_{a}=0, \mathbf{k}_{3}=0$ and $\mathbf{k}_{1}+\mathbf{k}_{2}=0$ respectively in these cases, the parametrized formulas for the invariant fields are valid for structure constant tensor components in arbitrary standard diagonal form for all the Bianchi types. We shall only evaluate them at the canonical components for each Bianchi type. The Bianchi Types I, II, 
and III may also be imbedded in $\operatorname{GL}(3, R)^{+}$by exponentiating the following canonical bases as above in the respective cases:

I. $\left\{\mathbf{e}_{1}^{1}, \mathbf{e}_{2}{ }_{2}, \mathbf{e}^{3}{ }_{3}\right\}$, II. $\left\{\mathbf{e}_{1}^{3}, \mathbf{e}_{2}^{3},-\mathbf{e}_{1}\right\}$, III. $\left\{\mathbf{e}_{1}^{2}+\mathbf{e}_{2}{ }_{2},-\mathbf{e}^{3}{ }_{1}+\mathbf{e}^{2}{ }_{2}, 2 \mathbf{e}^{1}{ }_{1}\right\}$.

From now on we will restrict our attention to canonical components for each Bianchi type. The canonical adjoint $\operatorname{group}_{\operatorname{Ad}_{e}}(G)$ for Type I is trivial while it is two-dimensional for Types II and III with respective bases $\left\{\mathbf{k}_{1}, \mathbf{k}_{2}\right\}$ and $\left\{\mathbf{k}_{1}, \mathbf{k}_{3}\right\}$ of the corresponding Lie algebras. The Class A adjoint groups are automatically unimodular since the matrices $\left\{\mathbf{k}_{a}\right\}$ are traceless while in the Class B case the matrices $\left\{\mathbf{k}_{1}, \mathbf{k}_{2}\right\}$ are a basis of the canonical special adjoint Lie algebra $\mathfrak{s a d}(\mathfrak{g})$. The canonical automorphism groups $A_{u}(\mathfrak{g})$ and special automorphism groups SAut $(g)$ are easily obtained by inspection of the transformation equations for $\left\{n^{a b}, a_{c}\right\}$ ignoring discrete automorphisms, i.e. we will only discuss their components connected to the identity. For Types VIII and IX the canonical adjoint and automorphism groups coincide and are respectively $\mathrm{SO}(2,1)$ and $\mathrm{SO}(3, R)$. For the remaining nondegenerate types and $V I_{-1 / 9}$, $\mathbf{n}$ has rank two and the only additional automorphisms are generated by $\mathbf{I}^{(3)}$. Either $\left\{\mathbf{k}_{1}, \mathbf{k}_{2}, \mathbf{k}_{3}, \mathbf{I}^{(3)}\right\}$ or $\left\{\mathbf{k}_{1}, \mathbf{k}_{2}, \mathbf{k}_{3}^{0}\right.$, $\left.\mathbf{I}^{(3)}\right\}$ is a basis of ant $_{e}(\mathfrak{g})$. The first three elements of the first basis span ad $(\mathfrak{g})$ while the first three of the latter span saut $(\mathfrak{g}) ;\left\{\mathbf{k}_{1}, \mathbf{k}_{2}\right\}$ or equivalently $\left\{\mathbf{e}^{3}{ }_{1}, \mathbf{e}^{3}{ }_{2}\right\}$ is a basis of $\mathfrak{s a d}_{e}(\mathrm{~g})$. For the two Cases II and IV for which $\mathbf{n}$ has rank one, assume $n^{(a)}$ $=\delta^{a}{ }_{1}$ so they can be discussed simultaneously. Invariance of $\mathbf{n}$ under the action of $A \in \mathrm{GL}(3, \mathbf{R})$ imposes the condition $A^{a}{ }_{1}=\delta^{a}{ }_{1}|\operatorname{det} \mathbf{A}|^{1 / 2}$ yielding for the Class A Type II a six-dimensional automorphism group whose unimodular subgroup is the fivedimensional subgroup of $\operatorname{SL}(3, R)$ whose elements satisfy $A^{a}{ }_{1}=\delta^{a}{ }_{1}$. For Type $I V$, the invariance of $a_{b}=\delta_{b}^{3}$ in addition forces $A_{1}^{1}=A_{2}^{2}>0$ by the previous condition, resulting in a four-dimensional automorphism group again generated by either $\left\{\mathbf{k}_{1}, \mathbf{k}_{2}, \mathbf{k}_{3}^{0}, \mathbf{I}^{(3)}\right\}$ or $\left\{\mathbf{e}^{3}{ }_{1}, \mathbf{e}^{3}{ }_{2}, \mathbf{k}_{3}^{0}, \mathbf{I}^{(3)}\right\}$ and whose unimodular subgroup SAut $_{e}(\mathfrak{g})$ has $A^{1}{ }_{1}=A^{2}{ }_{2}=1$. The latter group is generated by $\left\{\mathbf{e}^{3}{ }_{1}, \mathbf{e}^{3}{ }_{2}, \mathbf{k}_{3}^{0}\right\}$ which happens to coincide with the above matrix realization of the Type II group. In the Type $\mathrm{V}$ case, invariance of $a_{b}=\delta^{3}{ }_{b}$ requires only $A^{3}{ }_{a}=\delta^{3}{ }_{a}$ resulting in a sixdimensional canonical automorphism group with a five-dimensional special automorphism subgroup. In the trivial Type I case, $\operatorname{GL}(3, \mathbf{R})$ and $\operatorname{SL}(3, R)$ are the automorphism and special automorphism groups respectively.

The first application of our explicit knowledge of the canonical automorphism group Aut $_{e}(\mathfrak{g})$ for each Bianchi type will be to study $\mathscr{M}_{\text {true }}(G)$ for each type via the action of $A_{u}(\mathfrak{g})$ on the six-dimensional manifold $\mathscr{M}$. Let $\mathscr{M}_{D} \subset \mathscr{M}$ and $\overline{\mathscr{M}}_{D} \subset \overline{\mathscr{M}}$ be the diagonal submanifolds of $\mathscr{M}$ and $\overline{\mathscr{M}}$ respectively. It turns out that for the way we have chosen canonical components, $\mathscr{M}_{D}$ or a submanifold of it may be used as a local slice for this action. Let the Taub submanifold $\mathscr{M}_{T(1)} \subset \mathscr{M}_{D}$ be the submanifold of points $\mathbf{g} \in \mathscr{M}_{D}$ for which $g_{22}=g_{33}$ with $\mathscr{M}_{T(2)}$ and $\mathscr{M}_{T(3)}$ defined by cyclic permutation and $\overline{\mathscr{M}}_{T(a)}$ defined similarly. Finally let $\mathscr{M}_{I}$ be the "isotropic submanifold" of positive multiples of the identity matrix, projecting onto the single point $1 \in \tilde{\mathscr{M}}$. Any two of the Taub submanifolds intersect at $\mathscr{M}_{I}$.

Misner has introduced a useful parametrization of $\mathscr{M}_{D}$ adapted to the submanifolds $\mathscr{M}_{T(3)}$ and $\overline{\mathscr{M}}_{D}[9]$ :

$$
\begin{aligned}
& \mathscr{M}_{D} \ni \mathbf{g}=e^{2 \boldsymbol{\beta}} \quad \boldsymbol{\beta}=\beta^{A} \mathbf{e}_{A} \quad A, B=(0,+,-) \\
& \mathbf{e}_{0}=\mathbf{1} \quad \mathbf{e}_{+}=\operatorname{diag}(1,1,-2) \quad \mathbf{e}_{-}=\operatorname{diag}(\sqrt{3},-\sqrt{3}, 0) .
\end{aligned}
$$


The basis $\left\{\mathbf{e}_{A}\right\}$ is orthogonal with respect to the DeWitt trace inner product on $\operatorname{gl}(3, \mathbf{R})$ :

$$
\begin{aligned}
& \langle\mathbf{A}, \mathbf{B}\rangle_{D W}=\operatorname{Tr} \mathbf{A B}-\operatorname{Tr} \mathbf{A} \operatorname{Tr} \mathbf{B} \quad \mathbf{A}, \mathbf{B} \in \mathfrak{g l}(3, R) \\
& \left\langle\mathbf{e}_{A}, \mathbf{e}_{B}\right\rangle_{D W}=6 \eta_{A B}=6 \eta^{A B} \quad \boldsymbol{\eta}=\operatorname{diag}(-1,1,1) .
\end{aligned}
$$

Points of $\mathscr{M}_{T(3)}$ have $\beta^{-}=0$, while projection onto $\overline{\mathscr{M}}_{D}$ is accomplished by setting $\beta^{0}=0$ since $g=\operatorname{det} \mathbf{g}=e^{6 \beta^{0}}$.

For Type IX the orbits of $\operatorname{Aut}_{e}(\mathfrak{g})=S O(3, R)$ are transversal to $\mathscr{M}_{D}$ everywhere except on the Taub submanifolds where the orbit dimension degenerates from three to two except on their intersection $\mathscr{M}_{I}$ which is fixed under the action. For fixed $a$ the one-dimensional isotropy group on $\mathscr{M}_{T(a)}$ is generated by the matrix $\mathbf{k}_{a}=\operatorname{ad}_{e}\left(e_{a}\right)$ and corresponds to the additional left invariant Killing field $e_{a}$, while all three $e_{a}$ become Killing on $\mathscr{M}_{I}$ which corresponds to the bi-invariant metrics. For Type VIII the orbit dimension degenerates from three to two only on $\mathscr{M}_{T(3)}$, where the one-dimensional isotropy group generated by $\mathbf{k}_{3}=\operatorname{ad}_{e}\left(e_{3}\right)$ corresponds to the additional left invariant Killing vector field $e_{3}$. For both these types $\mathscr{M}_{D}$ is locally in a one-to-one correspondence with the orbits and $\overline{\mathscr{M}}_{D}$ therefore with the two dynamical degrees of freedom parametrized by $\beta^{+}$and $\beta^{-}$which describe the conformal 3-geometry.

For the remaining nondegenerate types, as well as the exceptional Type $V I_{-1 / 9}, \operatorname{Ad}_{e}(\mathfrak{g})$ acts transversally to $\mathscr{M}_{D}$ but $\operatorname{Aut}_{e}(\mathfrak{g})$ has an additional dimension corresponding to the matrix $\mathbf{I}^{(3)}=\frac{1}{3}\left(\mathbf{e}_{0}+2 \mathbf{e}_{+}\right)$which generates translations in the $\beta^{A}$ parameter space. This is a translation along $\beta^{+}$on $\overline{\mathscr{M}}_{D}$ so for these cases the submanifold $\beta^{+}=0$ is in a one-to-one correspondence with the orbits of $\operatorname{Aut}_{e}(\mathfrak{g})$ on $\overline{\mathscr{M}}$ and hence there is only one dynamical degree of freedom parametrized by $\beta^{-}$. For Types $V I I_{0}$ and $V I I_{h}$ the orbit dimension degenerates from four to three on $\mathscr{M}_{T(3)}$ where the isotropy group is generated by $\mathbf{k}_{3}=\operatorname{ad}_{e}\left(e_{3}\right)$ in the first case making $e_{3}$ a Killing vector field and by $\mathbf{k}_{3}^{0}$ in the second case corresponding to an additional Killing field $\xi$ in aut $(G)$. However, the full isotropy group $\mathscr{I}_{g}$ of $\mathscr{D}(G)$ on $\mathscr{M}_{T(3)}$ has dimension six since the corresponding Riemannian geometries $(G, g)$ are spaces of constant zero and negative curvature respectively.

For Type III the four-dimensional orbits of $\operatorname{Aut}_{e}(\mathfrak{g})$ are transversal to the submanifold $\beta^{+}=0$ of $\mathscr{M}_{D}$ so these geometries also have one degree of freedom corresponding to the parameter $\beta^{-}$. However, the full isotropy group for all Type III left invariant metrics is four-dimensional as shown by Bianchi. The submanifold $\beta^{-}=0$ of $\mathscr{M}_{D}$ corresponds to the negative curvature analogues of the Kantowski-Sachs geometries which have no dynamical degrees of freedom [11].

For Types II and V the six-dimensional group Aut $_{e}(\mathfrak{g})$ is transitive on $\overline{\mathscr{M}}$ with a one-dimensional isotropy group which at the identity is generated by $\mathbf{e}^{1}{ }_{2}-\mathbf{e}^{2}{ }_{1}$ in both cases. Therefore all Type II and V left invariant metrics are isometric to within a conformal constant and so have no dynamical degrees of freedom. However, all Type $\mathrm{V}$ geometries are spaces of constant negative curvature and have two additional Killing vector fields not belonging to aut $(G)$ which vary on $\mathscr{M}$. For Type I, Aut $(\mathfrak{g})=\mathrm{GL}(3, \mathbf{R})$ is transitive on $\mathscr{M}$ itself so all Type I metrics are isometric. This is obvious since all Type I metrics are flat; the additional Killing fields all lie in $\operatorname{aut}(G)$. Note that for Types II and V, the orbits of the action of 
Aut $_{e}(\mathrm{~g})$ on $\mathscr{M}$ are in a one-to-one correspondence with the isotropic submanifold $\mathscr{M}_{I}$ of $\mathscr{M}_{D}$ for which $\beta^{+}=\beta^{-}=0$, while in the Type I case the orbit of the single point $1 \in \mathscr{M}_{D}$ for which $\beta^{A}=0$ is $\mathscr{M}$ itself.

\section{Spatially Homogeneous Spacetimes and ADM: Kinematics}

Recall that if $X(t)$ is a parametrized curve in aut $(G)$, i.e. a time-dependent vector field on $G$, its flow is a curve $h(t)$ in $\operatorname{Aut}(G)$ which maps onto a curve in $\operatorname{Aut}(g)$. The action of $h(t)$ on $\mathfrak{g}$ and $\mathscr{M}(G)$ by dragging along is then equivalent to the action on these spaces of the latter curve and we obtain a family of curves in $\mathfrak{g}$ and $\mathscr{M}(G)$ (the orbits), i.e. time-dependent left invariant vector fields and metrics on $G$. Aut $(G)$ and $\operatorname{aut}(G)$ are the minisuperspace analogues of $\mathscr{D}(G)$ and $\mathfrak{X}(G)$ in the full theory of general relativity. We will essentially be describing some of the ideas of the geometric approach of Fischer and Marsden [2] in this finite-dimensional example.

Consider the manifold $M=R \times G$ where $G$ is a simply connected three-dimensional Lie group. A choice of basis $e$ of $g$ together with the natural coordinate vector field $\partial / \partial t$ on $R$ via many identifications common with product manifolds induces a global frame $\left\{\partial / \partial t, e_{a}\right\}$ on $M$ with dual frame $\left\{d t, \omega^{a}\right\} .\left\{\tilde{e}_{a}\right\}$ and $\tilde{\mathfrak{g}}$ itself may also be interpreted as vector fields on $M$. Note that

$$
\left[\partial / \partial t, e_{a}\right]=0=\left[\partial / \partial t, \tilde{e}_{a}\right]
$$

$G$ acts naturally on $R \times G$ on the left, with orbits $G_{t}=\{t\} \times G, t \in R$ and with $\tilde{\mathfrak{g}} \subset \mathfrak{X}(M)$ the Lie algebra of generators of the action. Any tensor field on $M$ which is invariant uner this action has components in the frame $\left\{\partial / \partial t, e_{a}\right\}$ which depend only on $t$ and will be called spatially homogeneous (SH). The frame itself is an example of a $\mathrm{SH}$ frame on $M$.

A SH spacetime $\left(M,{ }^{4} g\right)$ is such a manifold with a SH Lorentzian metric ${ }^{4} g$ such that the "hypersurfaces of homogeneity" $G_{t}$ are spacelike for $t$ in some open interval about zero. We will only be interested in that part of the spacetime in which the latter condition is satisfied. $\tilde{\mathfrak{g}} \subset \mathfrak{X}(M)$ is a Lie algebra of Killing vector fields of ${ }^{4} \mathrm{~g}$. Let us assume that $\partial / \partial t$ is a field of future pointing $\mathrm{SH}$ normals to the $\mathrm{SH}$ foliation of $M$ but not necessarily a unit vector field. Then $\partial / \partial t=N e_{\perp}$, where $N$ is a curve in $R$ (i.e. a function of $t$ only) and $e_{\perp}$ is the field of future pointing unit normals to the $\mathrm{SH}$ foliation of $M$.

The metric may then be expressed in the frame $\left\{\partial / \partial t, e_{a}\right\}$ as follows [10]:

$$
{ }^{4} g=-N^{2} d t \otimes d t+g_{a b} \omega^{a} \otimes \omega^{b},
$$

where the lapse function $N$ is a curve in $R$, the shift vector field vanishes and $\mathbf{g}=g_{a b} \mathbf{e}_{a}^{b}$ is a curve in $\mathscr{M} \cdot g=g_{a b} \omega^{a} \otimes \omega^{b}$ (actually its restriction to $G_{t}$ ) may be interpreted as a curve in $\mathscr{M}(G)$ or a time-dependent left invariant metric on $G$. This will remain true as long as the shift vector field is a curve confined to aut $(G)$. Let us extend the terminology $\mathrm{SH}$ to any curve of left invariant tensor fields on $G$ corresponding to a tensor field defined within each of the spatially homogeneous hypersurfaces. The extrinsic curvature of these hypersurfaces as well as the induced metric on them are examples. Any $\mathrm{SH}$ tensor field on $M$, when decomposed into 
perpendicular and parallel parts with respect to the $\mathrm{SH}$ foliation, induces a collection of such fields, each of which may be interpreted as a time-dependent left invariant field on $G$.

$G_{0}$ will be viewed as the initial value surface in our ADM approach. We want to use a SH lapse in order to take advantage of the natural slicing of the spacetime and a shift vector field confined to $\operatorname{aut}(G)$ in order that the induced metric on the $\mathrm{SH}$ foliation be a curve in $\mathscr{M}(G)$. The latter space will play the role of the "miniconfiguration space" of the dynamics of SH cosmology. Suppose $\partial / \partial \bar{t}$ $=\partial / \partial t+\vec{N}$, with $\vec{N}=N^{a} e_{a}$ a curve in $\operatorname{aut}(G)$. We may complete $\partial / \partial \bar{t}$ to a frame on $M$ adapted to the natural slicing by dragging along the "reduced frame" $\left\{e_{a}\right\}$ on $G_{0}$ to each hypersurface $G_{t}$ using the flow of $\partial / \partial \bar{t}$ to obtain a SH frame $\left\{\partial / \partial \bar{t}, \bar{e}_{a}\right\}$ with dual frame $\left\{d t, \bar{\omega}^{a}\right\}$ :

$$
\begin{aligned}
& \bar{e}_{a}=S_{a}^{-1 b} e_{b} \quad \bar{\omega}^{a}=S^{a}{ }_{b}\left(\omega^{b}-N^{b} d t\right) \\
& { }_{g}=-N^{2} d t \otimes d t+\bar{g}_{a b}\left(\bar{\omega}^{a}+\bar{N}^{a} d t\right) \otimes\left(\bar{\omega}^{b}+\bar{N}^{b} d t\right) .
\end{aligned}
$$

Here $\mathbf{S}$ is a curve in Aut $_{e}(\mathfrak{g})$ passing through the identity at $t=0, \bar{N}^{a}=S^{a}{ }_{b} N^{b}$ are the components of the shift vector field in the new frame and $\overline{\mathbf{g}}=f_{\mathbf{S}}(\mathbf{g})$ is another curve in $\mathscr{M}$. The restriction to $\mathrm{SH}$ shift vector fields has the effect of confining the curve $\mathbf{S}$ to the adjoint group $\operatorname{Ad}_{e}(G)$. Note that due to the properties of dragging along:

$$
\left[\bar{e}_{a}, \bar{e}_{b}\right]=C_{a b}^{c} \bar{e}_{c} \quad\left[\partial / \partial \bar{t}, \bar{e}_{a}\right]=0 .
$$

The latter condition is called the comoving condition. Any comoving SH frame $\left\{\partial / \partial \bar{t}, \bar{e}_{a}\right\}$ such that $\left\{\bar{e}_{a}\right\}$ are tangent to the SH foliation might be called a SH comoving ADM frame adapted to the SH foliation. With each such frame is associated a product structure for $M$ similar to $R \times G$ and a class of local coordinate systems $\left\{\bar{t}, \bar{x}^{a}\right\}$ on $M$ where $\left\{\bar{x}^{a}\right\}$ are dragged along from local coordinates $\left\{x^{a}\right\}$ on $G_{0}$ which may be identified with $G$. The usual discussions of dynamics rely on such ADM coordinates.

Let $\left\{\mathbf{E}_{i}\right\}$ be a basis of aut $(\mathfrak{g})$ and $\left\{W^{i}\right\}$ and $\left\{\tilde{W}^{i}\right\}$ the corresponding bases of the respectively left and right invariant 1 -forms induced on Aut $_{e}(\mathfrak{g})$. Then

$$
\mathbf{S}^{-1} \dot{\mathbf{S}}=\mathbf{E}_{i} \dot{W}^{i}, \quad \dot{\mathbf{S}} \mathbf{S}^{-1}=\mathbf{E}_{i} \dot{W}^{i}
$$

where $\dot{\mathbf{S}}=d / d t \mathbf{S}$ and $\dot{W}^{i}=W^{i}\left(S^{\prime}\right)$ and $\dot{\tilde{W}}^{i}=W^{i}\left(S^{\prime}\right)$ are the components of the tangent $S^{\prime}$ to the curve $\mathbf{S}$ with respect to the corresponding left and right invariant frames on $\operatorname{Aut}_{e}(\mathfrak{g})$. Expressing the comoving condition $\left[\partial / \partial \bar{t}, \bar{e}_{a}\right]=0$ in terms of the original frame leads to the following equivalent equations:

$$
\mathbf{A}=\mathbf{E}_{i} \dot{W}^{i}, \quad \overline{\mathbf{A}}=\mathbf{E}_{i} \dot{\tilde{W}}^{i}
$$

where $\mathbf{A}=\operatorname{ad}_{e}(\vec{N})$ and $\overrightarrow{\mathbf{A}}=\operatorname{ad}_{\bar{e}}(\vec{N})=\mathbf{S A S} \mathbf{S}^{-1}$ are curves in aut $(\mathfrak{g})$ generated by the curve $\vec{N}$ in aut $(G)$ acting on $\mathfrak{g}$. When $\vec{N}=N^{a} e_{a}=\bar{N}^{a} \bar{e}_{a}$ is $\mathrm{SH}$, we have instead:

$$
\mathbf{S}^{-1} \dot{\mathbf{S}}=\mathbf{k}_{a} \dot{\omega}^{a}, \quad \dot{\mathbf{S}} \mathbf{S}^{-1}=\mathbf{k}_{a} \dot{\tilde{\omega}}^{a}, \quad \mathbf{A}=N^{a} \mathbf{k}_{a}, \quad \overline{\mathbf{A}}=\bar{N}^{a} \mathbf{k}_{a},
$$

so these reduce to $N^{a}=\dot{\omega}^{a}$ and $\bar{N}^{a}=\dot{\tilde{\omega}}$. (Other solutions are possible when $\left\{\mathbf{k}_{a}\right\}$ are not linearly independent matrices. For example if $\mathbf{k}_{a}=0$, an arbitrary SH shift is possible with $\mathbf{S}=\mathbf{1}$.) 
In addition to the usual choice of zero shift, one may choose the shift vector field to satisfy the minimal distortion equation (MDE) of Smarr and York [11]. To consider this equation we must first study the covariant differentiation associated with a metric $y=g_{a b} \omega^{a} \otimes \omega^{b} \in \mathscr{M}(G)$. (We assume that the basis $e$ of $g$ is canonical.) As is well known [10], the components $\Gamma_{b c}^{a}=\omega^{a}\left(\nabla e_{b} e_{c}\right)$ of the metric connection $\nabla$ in the frame $e$ are given by

$$
\Gamma_{b c}^{a}=\frac{1}{2} C_{b c}^{a}+K_{b c}^{a},
$$

where $K_{b c}^{a}=C_{(b c)}^{a}$ and indices are raised and lowered by means of $\mathbf{g}$ and its inverse $\mathbf{g}^{-1}=g^{a b} \mathbf{e}^{b}$. Let

$$
\mathbf{K}_{a}=K_{a c}^{b} \mathbf{e}_{b}^{c}=\frac{1}{2}\left(\mathbf{k}_{a}+\mathbf{g}^{-1} \mathbf{k}_{a}{ }^{T} \mathbf{g}\right) .
$$

These are just the symmetrizations with respect to $\mathbf{g}$ of the adjoint matrices $\mathbf{k}_{a}$, namely $K_{a b c}=k_{a(b c)}=-\frac{1}{2}\left(t_{e_{a}} \mathscr{y}\right)_{b c}$.

The components of the curvature tensor and its various contractions may be obtained by evaluating standard formulas [10] which simplify since $d \Gamma^{a}{ }_{b c}=0$. For example, the Ricci tensor components $R^{a}{ }_{b}=R^{a c}{ }_{b c}$, the scalar curvature $R$ and the Einstein tensor components $G^{a}{ }_{b}=R^{a}{ }_{b}-\frac{1}{2} \delta^{a}{ }_{b} R$ can be written [12]:

$$
\begin{aligned}
\mathbf{R}= & R^{a}{ }_{b} \mathbf{e}^{b}{ }_{a}=2 \mathbf{m}^{2}-\mathbf{m} \operatorname{Tr} \mathbf{m}-\left(\operatorname{Tr}^{2}-\frac{1}{2} \operatorname{Tr}^{2} \mathbf{m}\right) \mathbf{1}-2 a^{a} a_{b} \mathbf{e}_{a}^{b}-2 a^{c} \mathbf{K}_{c} \\
R= & \operatorname{Tr} \mathbf{R}=-\left(\operatorname{Tr} \mathbf{m}^{2}-\frac{1}{2} \operatorname{Tr}^{2} \mathbf{m}\right)-6 a^{c} a_{c} \\
\mathbf{G}= & G^{a}{ }_{b} \mathbf{e}^{b}{ }_{a}=2 \mathbf{m}^{2}-\mathbf{m} \operatorname{Tr} \mathbf{m}-\frac{1}{2}\left(\operatorname{Tr}^{2}-\frac{1}{2} \operatorname{Tr}^{2} \mathbf{m}-6 a^{c} a_{c}\right) \mathbf{1} \\
& -2 a^{a} a_{b} \mathbf{e}^{b}{ }_{a}-2 a^{c} \mathbf{K}_{c},
\end{aligned}
$$

where $\mathbf{m}=g^{-1 / 2} \mathbf{n g}$, while the curvature tensor components are

$$
R^{a b}{ }_{c d}=-\delta_{c d g}^{a b f} G_{f}^{g} .
$$

If $\xi \in \mathfrak{X}(G)$ let us call the Lie derivative

$$
t_{\xi} g=2 \nabla_{(a} \xi_{b)} \omega^{a} \otimes \omega^{b}
$$

the Killing derivative of $\xi$ and its traceless part the conformal Killing derivative of $\xi[5]$ :

$$
(L \xi)_{a b}=2\left(\nabla_{(a} \xi_{b)}-\frac{1}{3} g_{a b} \nabla_{c} \xi^{c}\right) .
$$

Let $\mathbf{A}=\operatorname{ad}_{e}(\xi)$ and let $\mathbf{A}^{\#}$ be its symmetrization with respect to $\mathbf{g}$, namely $A_{b}^{\# a}$ $=g^{a c} A_{(c b)}$. A brief calculation shows that

$$
\begin{aligned}
& \nabla_{(a} \xi_{b)}=-A_{(a b)} \quad \nabla_{a} \xi^{a}=-\operatorname{Tr} \mathbf{A}^{\#}=-\operatorname{Tr} \mathbf{A} \\
& \nabla_{b} \xi^{a}+\nabla^{a} \xi_{b}=-2 A^{\# a}{ }_{b} \quad(L \xi)^{a}{ }_{b}=-2\left(A^{\# a}{ }_{b}-\frac{1}{3} \delta^{a}{ }_{b} \operatorname{Tr} A^{\#}\right) .
\end{aligned}
$$

For $\xi \in \operatorname{aut}(G), \mathbf{A}$ and $\mathbf{A}^{\#}$ are constant matrices so the Killing and conformal Killing derivatives of $\xi$ are left invariant.

Let $T_{b}^{a}$ be the constant mixed components of a left invariant symmetric second rank tensor or tensor density $T$ on $G$ and let $\mathbf{T}=T_{b}^{a} \mathbf{e}^{b}{ }_{a}$. Since $\Gamma_{a c}^{c}=0$ the divergence of either the tensor or the tensor density is given by the single formula:

$$
\nabla_{b} T_{a}^{b}=\operatorname{Tr} \mathbf{k}_{a} \mathbf{T}-2 a_{b} T_{a}^{b}=\operatorname{Tr} \mathbf{k}_{a} \mathbf{T}-2 a T_{a}^{3} .
$$


In particular, if $\mathbf{T}=T^{A} \mathbf{e}_{A}$, then $\nabla_{b} T_{a}^{b}=6 a T^{+} \delta^{3}{ }_{a}$. Since the unit tensor (components $\delta^{a}{ }_{b}$ ) is divergenceless, the divergence of $T$ agrees with the divergence of any tensor differing from it by a constant multiple of the unit tensor ${ }^{2}$.

Returning to the cosmological situation, we can now easily evaluate the components of the extrinsic curvature in the barred frame (also a canonical frame on $G$ for each $t$ ) $[10]$ :

$$
\begin{aligned}
& 2 N \bar{K}_{a b}=-\dot{\bar{g}}_{a b}+2 \bar{\nabla}_{(a} \bar{N}_{b)} \\
& 2 N \overline{\mathbf{K}}=2 N \bar{K}_{b}^{a} \mathbf{e}_{a}^{b}=-\overline{\mathbf{g}}^{-1} \dot{\overline{\mathbf{g}}}-2 \overline{\mathbf{A}}^{\#} .
\end{aligned}
$$

Let us assume that we pick the shift $\vec{N}$ so that it generates the curve $\mathbf{S}$ which transforms the curve $\mathbf{g}$ back to the submanifold of $\mathscr{M}_{D}$ which we found to be in a one-to-one correspondence (locally) with the orbits of $A_{u t}(\mathfrak{g})$ on $\mathscr{M}$. The resulting curve $\overline{\mathbf{g}}=f_{\mathbf{s}}(\mathbf{g})$ will then lie in this submanifold so we may write

$$
\begin{aligned}
& \overline{\mathbf{g}}=e^{2 \overline{\boldsymbol{\beta}}}, \quad \overline{\mathbf{g}}^{-1} \dot{\overline{\mathbf{g}}}=2 \dot{\overline{\boldsymbol{\beta}}} \\
& 2 N \overline{\mathbf{K}}=-2 \dot{\overline{\boldsymbol{\beta}}}-2 \overline{\mathbf{A}}^{\#} .
\end{aligned}
$$

The minimal distortion equation is

$$
\bar{\nabla}_{b}(\overrightarrow{L N})^{b}{ }_{a}=\bar{\nabla}_{b}\left(2 N\left(\bar{K}_{a}^{b}-\frac{1}{3} \delta_{a}^{b} \operatorname{Tr} \overline{\mathbf{K}}\right)\right)=2 N \bar{\nabla}_{b} \bar{K}_{a}^{b},
$$

but

$$
\begin{aligned}
& \overline{(L \vec{N}}_{b}^{a}=-2\left(\bar{A}_{b}^{\# a}-\frac{1}{3} \delta_{b}^{a} \operatorname{Tr} \overline{\mathbf{A}}^{\#}\right) \quad \bar{\nabla}_{b} \overline{(L \vec{N})_{b a}}=-2 \bar{\nabla}_{b} \bar{A}_{a}^{\# b} \\
& 2 N \bar{\nabla}_{b} \bar{K}_{a}^{b}=-6 a \dot{\bar{\beta}}^{+} \delta_{a}^{3}-2 \bar{\nabla}_{b} \bar{A}_{a}^{\# b} .
\end{aligned}
$$

However, when $a$ is nonzero, $\bar{\beta}^{+}$is a kinematical degree of freedom which we assumed was transformed away by $\mathbf{S}$, i.e. $\bar{\beta}^{+}=0$. The shift vector field $\vec{N}$ is thus a solution of the MDE. It is unique up to solutions of the homogeneous equation, i.e. up to a curve in $\operatorname{aut}(G)$ whose Killing derivative is divergenceless.

For all the nondegenerate types except $\mathrm{VI}_{0}$ and $\mathrm{VII}_{0}$, as well as Type $\mathrm{III}$ and Type VI ${ }_{-1 / 9}$, no such solutions exist which are not identically zero, but for Types $\mathrm{VI}_{0}$ and $\mathrm{VII}_{0}$ the vector field $\xi \in \mathfrak{a u t}(G)$ corresponding to $\operatorname{ad}_{e}(\xi)=\mathbf{I}^{(3)}$ has a divergenceless Killing derivative as long as $\mathbf{g} \in \mathscr{M}_{D}$. This means that in this case $\bar{\beta}^{+}$ need not be transformed to zero in order to make the shift a solution of the MDE, as is clear from the above anyway. Only Types I, II, and V remain. For Type I $\operatorname{aut}(G)$ is automatically "Killing divergenceless" and the extrinsic curvature is automatically divergenceless, so any curve in aut $(G)$ is a solution of the MDE. Let $\mathscr{M}_{S(1)}$ be the ("symmetric case") submanifold of for which $g_{23}=g_{32}$ are the only nonvanishing off-diagonal components of $\mathbf{g}$ and define $\mathscr{M}_{S(2)}$ and $\mathscr{M}_{S(3)}$ in a cyclic manner, with similar definitions for $\overline{\mathscr{M}}_{S(a)}$. A basis for aut $e_{e}(\mathrm{~g})$ for Type II is $\left\{\mathbf{k}_{1}, \mathbf{k}_{2}\right.$, $\left.\mathbf{e}^{1}{ }_{2}, \mathbf{e}^{2}{ }_{1}, \mathbf{e}^{1}{ }_{1}-\mathbf{e}^{2}{ }_{2}, \mathbf{e}^{1}{ }_{1}+\mathbf{e}^{2}{ }_{2}+2 \mathbf{e}^{3}{ }_{3}\right\}$ and for Type $\mathrm{V}\left\{\mathbf{k}_{1}, \mathbf{k}_{2}, \mathbf{k}_{3}, \mathbf{e}^{1}{ }_{2}, \mathbf{e}^{2}{ }_{1}, \mathbf{e}^{1}{ }_{1}-\mathbf{e}^{2}{ }_{2}\right\}$ is such a basis. The last four elements of the first basis and the last three of the second basis correspond to elements of aut $(G)$ with divergenceless Killing derivative as long as $\mathbf{g} \in \mathscr{M}_{\mathrm{S}(3)}$. In other words the minimal distortion shift in these cases is

2 For this reason the minimal strain and minimal distortion shift equations of Smarr and York coincide in our situation, provided that the shift is confined aut $(G)$ 
defined only up to a curve in aut $(G)$ which has the effect of allowing $\overline{\mathbf{g}}$ to wander on $\mathscr{M}_{S(3)}$. These observations have important dynamical effects.

\section{Dynamics}

So far we have only discussed kinematical considerations. However, what we have learned can dramatically simplify the dynamics. Assume the unbarred frame of the previous section and set the lapse to unity, no restriction since we can easily reparametrize the time whenever we wish. $\partial / \partial t=e_{\perp}$ is then the unit normal to the $\mathrm{SH}$ foliation; let $\omega^{\perp}=d t$ and let Greek indices assume the values $\{\perp, 1,2,3\}$. Let $T_{\beta}^{\alpha}$ be the components in the frame $\left\{e_{\alpha}\right\}$ of the energy-momentum tensor of a spatially homogeneous matter source. The matter variables and their equations of motion will be ignored since it is the geometrical equations we want to focus on. The Einstein equations

$$
G_{\beta}^{\alpha}=\kappa T_{\beta}^{\alpha}
$$

for the component functions $g_{a b}(t)$ in this frame are equivalent to the following driven Lagrangian or Hamiltonian systems with configuration space $\mathscr{M}$, velocity phase space $T \mathscr{M}$ and momentum phàse space $T \cdot \mathscr{M}^{*}$, subject to four constraints $[12]^{3}$ :

$$
\begin{aligned}
& L_{G}(\mathbf{g}, \dot{\mathbf{g}})=g^{1 / 2}\langle\mathbf{K}, \mathbf{K}\rangle_{D W}+g^{1 / 2} R \quad \mathbf{K}=-\frac{1}{2} \mathbf{g}^{-1} \dot{\mathbf{g}} \\
& \delta L_{G} / \delta g_{a b}=\partial L_{G} / \partial g_{a b}-\left(\partial L_{G} / \partial \dot{g}_{a b}\right)^{\prime}=-Q^{a b}-\kappa g^{1 / 2} T^{a b} \\
& E_{G}(\mathbf{g}, \mathbf{g})=g^{1 / 2}\langle\mathbf{K}, \mathbf{K}\rangle_{D W}-g^{1 / 2} R=2 \kappa g^{1 / 2} T^{\perp}{ }_{\perp} \\
& \operatorname{Tr} \mathbf{k}_{a} \mathbf{K}-2 a_{c} K_{a}^{c}=\kappa T^{\perp}{ }_{a} \\
& \pi^{a b}=\partial L_{G} / \partial \dot{g}_{a b} \quad \pi=\pi_{b}^{a} \mathbf{e}_{a}^{b}=-g^{1 / 2}(\mathbf{K}-\mathbf{1} \operatorname{Tr} \mathbf{K}) \\
& H_{G}(\mathbf{g}, \pi)=g^{-1 / 2}\langle\pi, \pi\rangle_{D W}^{*}-g^{1 / 2} R \\
& \dot{g}_{a b}=\left\{g_{a b}, H_{G}\right\} \quad \dot{\pi}^{a b}=\left\{\pi^{a b}, H_{G}\right\}+Q^{a b}+\kappa g^{1 / 2} T^{a b} \\
& \mathscr{H}^{a b} H_{G}-2 \kappa g^{1 / 2} T^{\perp}=0 \\
& \mathscr{H}_{a}^{G}=-2 \operatorname{Tr} \mathbf{k}_{a} \pi+4 a_{c} \pi_{a}^{c}=2 \kappa g^{1 / 2} T^{\perp} .
\end{aligned}
$$

We have used the notation

$$
\langle\mathbf{A}, \mathbf{B}\rangle_{D W}^{*}=\operatorname{Tr} \mathbf{A B}-\frac{1}{2} \operatorname{Tr} \mathbf{A} \operatorname{Tr} \mathbf{B} \quad \mathbf{A}, \mathbf{B} \in \mathfrak{g l}(3, R)
$$

for the dual of the DeWitt inner product while the matrix of mixed components $\mathbf{Q}=Q^{a}{ }_{b} \mathbf{e}^{b}$ is defined by

$$
\mathbf{Q}=2 g^{1 / 2}\left(a^{c} \mathbf{K}_{c}-2 a^{a} a_{b} \mathbf{e}_{a}^{b}\right) .
$$

3 Admittedly our notation indulges in many obvious identifications but this sloppiness is not only characteristic of classical mechanical discussions but extremely convenient 
The nonconservative force field $Q=Q^{a b} d g_{a b}=\operatorname{Tr}_{\mathbf{Q g}}{ }^{-1} d \mathbf{g}$ on $\mathscr{M}$ appears in the Class B case since the Einstein force field $G=-g^{1 / 2} G^{a b} d g_{a b}$ is not exact, as a simple calculation using the formulas of the previous section shows:

$$
\begin{aligned}
G & =-d U_{G}+Q \\
U_{G} & =-g^{1 / 2} R=\frac{1}{4} g^{-1 / 2}\langle\mathbf{n g}, \mathbf{n g}\rangle_{D W}^{*}+6 g^{1 / 2} g^{a b} a_{a} a_{b} .
\end{aligned}
$$

$U_{G}$ is the gravitational potential energy while the first term in either the Lagrangian or Hamiltonian is the kinetic energy, namely the inner product with itself in the DeWitt metric on $\mathscr{M}$ (apart from factors of two) of the velocity or momentum. The kinetic energy alone serves as a Lagrangian or Hamiltonian for the geodesics of the DeWitt metric on $\mathscr{M}$, which are explicitly known $[4,12]$. For perfect fluids and electromagnetic fields, matter variables can be chosen so that $U_{M}=-2 \mathrm{Kg}^{1 / 2} T^{\perp}{ }_{\perp}$ considered as a time-dependent potential energy function on $\mathscr{M}$ gives rise to the matter driving force [12]:

$$
\begin{array}{rlrl}
d U_{M} & =-\kappa g^{1 / 2} T^{a b} d g_{a b} \\
L & =L_{G}-U_{M} \quad & \delta L / \delta g_{a b}=-Q^{a b} \\
\mathscr{H} & =H_{G}+U_{M} \quad & \dot{\pi}^{a b}=\left\{\pi^{a b}, \mathscr{H}\right\}+Q^{a b} .
\end{array}
$$

In a certain sense $\operatorname{SAut}_{e}(\mathfrak{g})$ is a symmetry group of these systems. (The unimodularity condition is needed since densities are involved.) The gravitational potential energy $U_{G}$, the Einstein force field $G$ and the nonconservative force $Q$, for example, are invariant under the action of $\operatorname{SAut}_{e}(\mathrm{~g})$, as is the kinetic energy function on either $T \mathscr{M}$ or $T \mathscr{M}^{*}$ [which is invariant under the action of $\operatorname{SL}(3, R)$, the isometry group of the DeWitt metric on $\mathscr{M}]$. However, a symmetry of the Hamiltonian in the Class B case is insufficient to generate a constant of the motion, while the matter source may break the symmetry in either case. SAut $e^{(g)}$ can still be used to great advantage though.

In the nondegenerate case, to which we will restrict our attention until otherwise stated, SAut $(\mathfrak{g})$ is three-dimensional and its orbits on $\mathscr{M}$ are transversal to $\mathscr{M}_{D}$. This observation enables us to introduce a natural parametrization of the matrix of metric components adapted to the action of $\operatorname{SAut}_{e}(\mathfrak{g})$ on $\mathscr{M}$, namely

$$
\mathbf{g}=\mathbf{S}^{T} \mathbf{g}^{\prime} \mathbf{S}, \quad \mathbf{g}^{\prime}=e^{2 \boldsymbol{\beta}}=f_{\mathbf{S}}(\mathbf{g}) \in \mathscr{M}_{D}
$$

considered as a map from $\mathscr{M}_{D} \times \mathrm{SAut}_{e}(\mathrm{~g})$ onto $\mathscr{M}$ which is almost everywhere nonsingular (except where the orbit dimension degenerates). Using this map we can pull back our differential equations from $\mathscr{M}$ to $\mathscr{M}_{D} \times \mathrm{SAut}_{e}(\mathrm{~g})$ where they can be more easily studied.

For a given solution $\mathbf{g}(t)$ or equivalently $\left\{\mathbf{g}^{\prime}(t), \mathbf{S}(t)\right\}, \mathbf{g}^{\prime}(t)$ may be interpreted as the matrix of components of the metric with respect to a new reduced frame $e_{a}^{\prime}$ $=S^{-1 b}{ }_{a} e_{b}$ which can be completed to a SH comoving ADM frame $\left\{\partial / \partial t^{\prime}, e_{a}{ }^{\prime}\right\}$ on the spacetime corresponding to the introduction of some shift vector field which can be calculated from the equations of the previous section. For Types VIII and IX this coincides with the uniquely defined barred frame. For Types $\mathrm{VI}_{0}$ and $\mathrm{VII}_{0}$ the freedom in the minimal distortion shift permits a choice making it coincide with the barred frame, while in the nondegenerate Class B case the two frames are 
related by a scaling which removes $\beta^{+}$:

$$
\tilde{\mathbf{e}}_{a}=\mathbf{e}_{b}{ }^{\prime}\left(e^{-3 \beta^{+} \mathbf{I}^{(3)}}\right)_{a}^{b} .
$$

Our procedure will involve expressing all quantities of interest in terms of the primed frame.

A convenient basis of $\mathfrak{s a u t}_{e}(\mathfrak{g})$ for the nondegenerate Class A types is $\left\{\boldsymbol{\kappa}_{a}\right\}=\left\{\mathbf{k}_{a}\right\}$, while $\left\{\boldsymbol{\kappa}_{a}\right\}=\left\{\mathbf{e}^{3}{ }_{1}, \mathbf{e}^{3}{ }_{2}, \mathbf{k}_{3}^{0}\right\}$ will do for the nondegenerate Class B types. In the latter case the adjoint matrices are related by the following invertible transformation

$$
\mathbf{k}_{1}=a \boldsymbol{\kappa}_{1}-n^{(2)} \boldsymbol{\kappa}_{2}, \quad \mathbf{k}_{2}=n^{(1)} \boldsymbol{\kappa}_{1}+a \boldsymbol{\kappa}_{2}, \quad \mathbf{k}_{3}=\boldsymbol{\kappa}_{3}+a \mathbf{I}^{(3)} .
$$

Let $\mathscr{K}_{a}$ be the symmetrization of $\boldsymbol{\kappa}_{a}$ with respect to $\mathbf{g}^{\prime}=e^{2 \boldsymbol{\beta}}$ and define

$$
\mathscr{G}_{a b}=\operatorname{Tr} \mathscr{K}_{a} \mathscr{K}_{b}=\left\langle\mathscr{K}_{a}, \mathscr{K}_{b}\right\rangle_{D W} .
$$

The matrices $\left\{\boldsymbol{\kappa}_{a}\right\}$ and therefore $\left\{\mathscr{K}_{a}\right\}$ are off-diagonal and hence orthogonal to the diagonal matrices with respect to $\langle,\rangle_{D W}$; they are also orthogonal to each other so $\mathscr{G}_{a b}$ is diagonal and its inverse $\mathscr{G}^{a b}$ is also diagonal, with $\mathscr{G}^{a a}=\left(\mathscr{G}_{a a}\right)^{-1}$. Introducing the notation

$$
\begin{aligned}
& \beta=\operatorname{diag}\left(\beta^{1}, \beta^{2}, \beta^{3}\right) \quad \beta^{a b}=\beta^{a}-\beta^{b} \\
& \beta^{23}=3 \beta^{+}-\sqrt{3} \beta^{-} \quad \beta^{31}=-3 \beta^{+}-\sqrt{3} \beta^{-} \quad \beta^{12}=2 \sqrt{3} \beta^{-},
\end{aligned}
$$

then one finds that in the Class A case for each cyclic permutation $(a, b, c)$ of $(1,2,3)$

$$
\mathscr{G}_{a a}=\frac{1}{2}\left(n^{(b)} e^{\beta^{b c}}-n^{(c)} e^{-\beta^{b c}}\right)^{2} .
$$

When $\left\{n^{(b)}, n^{(c)}\right\}$ equals respectively $\{1,0\},\{1,-1\}$, and $\{1,1\}$ this expression may be written:

$$
\frac{1}{2} e^{2 \beta^{b c}}, \quad 2 \cosh ^{2} \beta^{b c}, \quad 2 \sinh ^{2} \beta^{b c} .
$$

In the Class B case the formula for $\mathscr{G}_{33}$ remains valid while $\mathscr{G}_{11}=\frac{1}{2} e^{2 \beta^{13}}$ and $\mathscr{G}_{22}=\frac{1}{2} e^{2 \beta^{23}}$.

The basis $\left\{\boldsymbol{\kappa}_{a}\right\}$ of saut $_{e}(\mathfrak{g})$ generates a basis for left and right invariant vector fields and 1-forms on $\mathrm{SAut}_{e}(\mathfrak{g})$ which for lack of symbols we will also designate by $\left\{e_{a}\right\},\left\{\tilde{e}_{a}\right\},\left\{\omega^{a}\right\}$, and $\left\{\tilde{\omega}^{a}\right\}$. (These are not be confused with the fields on $G$ or $R \times G$ having the same symbols.) The 1 -forms arise in the formulas

$$
\mathbf{S}^{-1} d \mathbf{S}=\boldsymbol{\kappa}_{a} \omega^{a} \quad d \mathbf{S} \mathbf{S}^{-1}=\boldsymbol{\kappa}_{a} \tilde{\omega}^{a} .
$$

Together with the $\left\{\beta^{A}\right\}$ coordinate fields on $\mathscr{M}_{D}$, these in turn induce fields on the product manifold $\mathscr{M}_{D} \times \mathrm{SAut}_{e}(\mathrm{~g})$ (again designated by the same symbols) and finally "coordinates" $\left\{\beta^{A}, \mathbf{S}, \dot{\beta}^{A}, \dot{\tilde{\omega}}^{a}\right\}$ and $\left\{\beta^{A}, \mathbf{S}, p_{A}, p_{a}\right\}$ on the tangent and cotangent bundles which correspond to taking components of tangent vectors and covectors in the frame $\left\{\partial / \partial \beta^{A}, \tilde{e}_{a}\right\}$ with dual frame $\left\{d \beta^{A}, \tilde{\omega}^{a}\right\}$. We have the corresponding formulas

$$
\mathbf{S}^{-1} \dot{\mathbf{S}}=\boldsymbol{\kappa}_{a} \dot{\omega}^{a} \quad \dot{\mathbf{S}} \mathbf{S}^{-1}=\boldsymbol{\kappa}_{a} \dot{\tilde{\omega}}^{a}
$$


A simple calculation yields the following expression for the primed components of the extrinsic curvature matrix, interpreted now as functions on the tangent bundle of $\mathscr{M}_{D} \times \mathrm{SAut}_{e}(\mathfrak{g})$

$$
\mathbf{K}^{\prime}=\mathbf{S K S}^{-1}=-\dot{\boldsymbol{\beta}}-\mathbf{K}_{a} \dot{\tilde{\omega}}^{a} .
$$

Since $\operatorname{det} \mathbf{S}=1, g=g^{\prime}=e^{6 \beta^{0}}$. The kinetic energy function on velocity phase space is therefore

$$
T=e^{3 \beta^{0}}\left\langle\mathbf{K}^{\prime}, \mathbf{K}^{\prime}\right\rangle_{D W}=e^{3 \beta^{0}}\left(6 \eta_{A B} \dot{\beta}^{A} \dot{\beta}^{B}+\mathscr{G}_{a b} \dot{\tilde{\omega}}^{a} \dot{\tilde{\omega}}^{b}\right) .
$$

The velocity-momentum relation is

$$
\begin{array}{ll}
p_{A}=\partial T / \partial \dot{\beta}^{A}=12 e^{3 \beta^{0}} \eta_{A B} \dot{\beta}^{B} & p_{a}=\partial T / \partial \dot{\tilde{\omega}}^{a}=2 e^{3 \beta^{0}} \mathscr{G}_{a b} \dot{\tilde{\omega}}^{b} \\
\dot{\beta}^{A}=\frac{1}{12} e^{-3 \beta^{0}} \eta^{A B} p_{B} & \dot{\tilde{\omega}}^{a}=\frac{1}{2} e^{-3 \beta^{0}} \mathscr{G}^{a b} p_{b} .
\end{array}
$$

Reexpressing $T$ in terms of the momenta, one obtains the kinetic energy function on momentum phase space:

$$
T=\frac{1}{4} e^{-3 \beta^{0}}\left(\frac{1}{6} \eta^{A B} p_{A} p_{B}+\mathscr{G}^{a b} p_{a} p_{b}\right) .
$$

The gravitational potential energy function is easily evaluated:

$$
\begin{aligned}
U_{G}= & \frac{1}{4} e^{3 \beta^{0}}\left\langle\mathbf{n} \mathbf{g}^{\prime}, \mathbf{n g}^{\prime}\right\rangle_{D W}^{*}+\mathbf{6} \mathbf{e}^{3 \beta^{0}} g^{\prime 33} a^{2} \\
= & \frac{1}{2} e^{\beta^{0}} V+6 a^{2} e^{\beta^{0}+4 \beta^{+}} \\
V= & e^{4 \beta^{+}}\left(n^{(1)} e^{2 \sqrt{3} \beta^{-}}-n^{(2)} e^{-2 \sqrt{3} \beta^{-}}\right)^{2}-2 n^{(3)} e^{-2 \beta^{+}}\left(n^{(1)} e^{2 V 3 \beta^{-}}\right. \\
& \left.\quad+n^{(2)} e^{-2 \sqrt{3} \beta^{-}}\right)+\left(n^{(3)}\right)^{2} e^{-8 \beta^{+}} .
\end{aligned}
$$

Similarly the primed components of the nonconservative force matrix are readily obtained:

$$
\mathbf{Q}^{\prime}=\mathbf{S} \mathbf{Q S} \mathbf{S}^{-1}=2 e^{3 \beta^{0}}\left(a^{\prime 3} \mathbf{K}_{3}^{\prime}-2 a^{\prime 3} a_{3} \mathbf{e}_{3}^{3}\right)=2 a e^{\beta^{0}+4 \beta^{+}}\left(a \mathbf{e}_{+}+\mathbf{K}_{3}\right) .
$$

The force field itself is then obtained from it as follows:

$$
\begin{aligned}
Q & =\operatorname{Tr} \mathbf{Q g}{ }^{-1} d \mathbf{g}=\operatorname{Tr}\left(\mathbf{Q}^{\prime}\left(2 d \boldsymbol{\beta}+2 \mathbf{K}_{a} \tilde{\omega}^{a}\right)\right)=4 a e^{\beta^{0}+4 \beta^{+}}\left(6 a d \beta^{+}+\mathscr{G}_{33} \tilde{\omega}^{3}\right) \\
Q_{+} & =24 a^{2} e^{\beta^{0}+4 \beta^{+}} \quad Q_{3}=4 a e^{\beta^{0}+4 \beta^{+}} \mathscr{G}_{33} .
\end{aligned}
$$

Finally we must evaluate the primed components of the velocity or momentum constraints. In the Class A case:

$$
\mathscr{H}_{a^{\prime}}^{G}=-e^{3 \beta^{0}} \mathscr{G}_{a b} \dot{\tilde{\omega}}^{b}=-\frac{1}{2} p_{a}=e^{3 \beta^{0}} \kappa T_{a^{\prime}}^{\perp},
$$

while in the Class B case:

$$
\begin{aligned}
& \mathscr{H}_{1^{\prime}}^{G}=e^{3 \beta^{0}}\left(3 a \mathscr{G}_{11} \dot{\tilde{\omega}}^{1}+n^{(2)} \mathscr{G}_{22} \dot{\tilde{\omega}}^{2}\right)=\frac{1}{2}\left(3 a p_{1}+n^{(2)} p_{2}\right)=e^{3 \beta^{0}} \kappa T^{\perp}{ }^{\prime}{ }^{\prime} \\
& \mathscr{H}_{2^{\prime}}^{G}=e^{3 \beta^{0}}\left(-n^{(1)} \mathscr{G}_{11} \dot{\tilde{\omega}}^{1}+3 a \mathscr{G}_{22} \dot{\tilde{\omega}}^{2}\right)=\frac{1}{2}\left(-n^{(1)} p_{1}+3 a p_{2}\right)=e^{3 \beta^{0}} \kappa T^{\perp}{ }_{2^{\prime}} \\
& \mathscr{H}_{3^{\prime}}^{G}=e^{3 \beta^{0}}\left(-6 a \dot{\beta}^{+}-\mathscr{G}_{33} \dot{\tilde{\omega}}^{3}\right)=-\frac{1}{2}\left(a p_{+}+p_{3}\right)=e^{3 \beta^{0}} \kappa T^{\perp}{ }_{3^{\prime}} .
\end{aligned}
$$

As is customary we are being sloppy about distinguishing functions on velocity or momentum phase space. These constraints may be solved for either the automorphism velocities or momenta. The Class A case is trivial:

$$
\dot{\tilde{\omega}}^{a}=-\mathscr{G}^{a b} \kappa T_{b^{\prime}}^{\perp} \quad p_{a}=-2 e^{3 \beta^{0}} \kappa T_{a^{\prime}}^{\perp},
$$


while in the Class B case:

$$
\begin{aligned}
& p_{1}=2 \kappa e^{3 \beta^{0}}\left(3 a T^{\perp}{ }_{1},-n^{(2)} T^{\perp}{ }^{\prime}\right) / Z \\
& p_{2}=2 \kappa e^{3 \beta^{0}}\left(n^{(1)} T^{\perp}{ }^{\prime}+3 a T^{\perp}{ }^{\prime}\right) / Z \\
& p_{3}=-a p_{+}-2 \kappa e^{3 \beta^{0}} T^{\perp}{ }^{\prime},
\end{aligned}
$$

with corresponding expressions for the velocities. For the exceptional case of Type $\mathrm{VI}_{-1 / 9}$, the quantity $Z=9 a^{2}+n^{(1)} n^{(2)}=\left(9+h^{-1}\right) a^{2}$ vanishes and $3 a=1=n^{(1)}$ $=-n^{(2)}$ so there are only two linearly independent constraints :

$$
\mathscr{H}_{1}^{G},=\frac{1}{2}\left(p_{1}-p_{2}\right)=-\mathscr{H}_{2}^{G},=e^{3 \beta^{0}} \kappa T^{\perp}{ }_{1},=-e^{3 \beta^{0}} \kappa T^{\perp}{ }^{\prime}{ }^{\prime},
$$

which explains why we have included it in the degenerate category.

As an aside we mention that $\vec{N}=\dot{\tilde{\omega}}^{a} e_{a^{\prime}}$ is the shift vector field associated with the primed frame in the Class A case, while in the Class B case it is

$$
\vec{N}=\left(a \dot{\tilde{\omega}}^{1}-n^{(2)} \dot{\tilde{\omega}}^{2}\right) e_{1^{\prime}}+\left(n^{(1)} \dot{\tilde{\omega}}^{1}+a \dot{\tilde{\omega}}^{2}\right) e_{2^{\prime}}+\dot{\tilde{\omega}}^{3}\left(e_{3^{\prime}}-a \xi^{\prime}\right) \text {. }
$$

The coefficients of $e_{1}$, and $e_{2}$, are the velocity components with respect to $\mathbf{k}_{1}$ and $\mathbf{k}_{2}$, while $e_{3^{\prime}}-a \xi^{\prime}$ is the dragged along vector field for which $\operatorname{ad}_{e^{\prime}}\left(e_{3^{\prime}}-a \xi^{\prime}\right)=\boldsymbol{\kappa}_{3}$.

By choosing the primed equivalents of the matter variables as new independent variables, the matrix $\mathbf{S}$ drops out of our classical mechanical system, leaving behind only the automorphism velocities or momenta both in the geometric and matter equations. The momentum constraints may then be used to determine the latter quantities in terms of the fluid variables and in the Class B case in terms of $\dot{\beta}^{+}$or $p_{+}$as well. Thus only three geometric degrees of freedom remain, subject to the energy constraint.

The energy constraint may be used to eliminate another degree of freedom by replacing the time $t$ as the integration variable by some function of $\boldsymbol{\beta}$, the usual and obvious choice being $\beta^{0}$, using a standard technique in classical mechanics equivalent to a new choice of lapse [13]. One solves the equation $\mathscr{H}+h=0$ for the momentum $p_{0}$ conjugate to $\beta^{0}$, defining

$$
-I_{h}=\mp\left(p_{+}{ }^{2}+p_{-}{ }^{2}+6\left(\eta^{a b} p_{a} p_{b}+4 e^{3 \beta^{0}}\left(U_{G}+U_{M}+h\right)\right)\right)^{1 / 2} .
$$

$I_{0}$ acts as the new Hamiltonian:

$$
d \beta^{ \pm} / d \beta^{0}=\left\{\beta^{ \pm}, I_{0}\right\} \quad d p_{ \pm} / d \beta^{0}=\left\{p_{ \pm}, I_{0}\right\}+\delta^{+}{ }_{ \pm} N Q_{+},
$$

while the lapse function is determined by

$$
N=d t / d \beta^{0}=\partial I_{h} /\left.\partial h\right|_{h=0}=12 e^{3 \beta^{0}} / I_{0} .
$$

The matter equations must be reparametrized using this lapse. The choice of the negative root for $-I_{h}$ makes $t$ an increasing function of $\beta^{0}$ (expansion) while the positive root makes $t$ a decreasing function of $\beta^{0}$ (contraction). Conventionally $\Omega=-\beta^{0}$ is taken as the integration variable [14]. This reduction technique is valid only on a segment of a solution curve for which $\beta^{0}$ is either strictly increasing or decreasing with $t$ and breaks down at points of maximum expansion.

The geometric degrees of freedom have therefore been reduced in number to two and these describe the conformal 3-geometry of the SH hypersurfaces. Even though we have called $\beta^{+}$a nondynamical or kinematical variable for all the 
nondegenerate types but VIII and IX, it is not possible to eliminate it from the dynamics. The reason for this is essentially that the minimal distortion shift requires four functions of time to specify it when it is unique [since aut $(G)$ is fourdimensional], whereas the minimal distortion equation can be used to determine at most only three of them, while in the case in which it is not unique, the one degree of freedom present in the minimal distortion shift is transferred to the dynamical variables if we allow the constraints to pick out a solution (the one which merely diagonalizes the matrix $\mathbf{g}$ ). The best one can do is determine a shift which diagonalizes the matrix of metric components, and this is exactly what the elimination of the special automorphism variables from our classical mechanical system corresponds to.

Motivated by other considerations, Ryan has used the canonical Type IX parametrization for all Bianchi types [14]. However, because $\operatorname{SO}(3, R)$ is compatible only with the Type IX case, this has prevented any practical application in the remaining types. In the Type IX case, the above scheme coincides with Ryan's [15].

Type III can be treated exactly as Type $\mathrm{VI}_{h}$ for $h \neq 0,-1$ or $-1 / 9$, the only difference being that the adjoint group trades off a dimension to the rest of the automorphism group and the shift vector field associated with the primed frame must be recalculated. The Type VI -1/9 $_{9}$ can also be treated using the same formulas up until the point where the constraints are used to determine the automorphism velocities or momenta, at which point only two of the four shift degrees of freedom can be pinned down, leaving at least three degrees of freedom remaining in the end.

This leaves Types I, II, and V which require individual treatment but have some similar features. Here the special automorphism group is too large and one has to arbitrarily choose a subgroup whose orbits are transversal to $\mathscr{M}_{D}$ to play the above game, provided that it is $\mathscr{M}_{D}$ to which one wants to attempt to reduce the geometric degrees of freedom. However, the degeneracy in the constraints for the first two types is an obstacle to the full reduction, while in the Type V case, $\mathscr{M}_{D}$ does not seem to be the appropriate submanifold to head for.

The choice of a particular three-dimensional subgroup of the larger special automorphism group also introduces problems. For example, if $\mathbf{g}(t)$ is a solution curve in $\mathscr{M}_{D}$, then $f_{\mathbf{A}^{-1}}(\mathbf{g}(t))$ is also a solution curve for every constant $\mathbf{A}$ in the special automorphism subgroup which leaves the matter potential invariant. Suppose A is a constant nondiagonal such matrix, but which doesn't belong to the subgroup we have chosen. Then in order to represent the curve $f_{\mathbf{A}-1}(\mathbf{g}(t))$ as $f_{\mathbf{S}^{-1}(t)}\left(\mathbf{g}^{\prime}(t)\right)$ where $\mathbf{S}(t)$ lies in the chosen subgroup and $\mathbf{g}^{\prime}(t)$ in $\mathscr{M}_{D}$, a complicated time dependence of the curve $\mathbf{S}(t)$ with corresponding additional complication for $\mathbf{g}^{\prime}(t)$ compared to $\mathbf{g}(t)$ is required to produce the trivial modification of the curve $\mathbf{g}(t)$. Furthermore, this trivial modification $f_{\mathbf{A}^{-1}}$ of the curve generates an isometric spacetime, and since we are only interested in the isometry classes of spacetimes, i.e. solution curves modulo the action of constant elements of the automorphism group, we do not care about such modifications. But we are stuck with fictitious degrees of freedom in attempting to extend the nondegenerate scheme to the degenerate cases. The way around this problem is to check whether or not arbitrary initial data can be obtained from diagonal initial data under the action of the automorphism group. If so we may simply ignore those degrees of freedom. 
Consider the Type I case. We might use the canonical Type IX parametrization, for example, but since the gravitational super-momentum is identically zero the automorphism degrees of freedom cannot be eliminated. However, one knows that as long as $U_{M}$ is invariant under $\operatorname{SL}(3, R)$ which is the case for perfect fluids, a solution curve $\mathbf{g}(t)$ projects down to a geodesic of the metric induced on $\overline{\mathscr{M}}$ by the DeWitt metric and so only the equations of motion for $g$ are nontrivial. From the point of view of the previous paragraph, initial data $\{\mathbf{g}, \dot{\mathbf{g}}\}$ can always be simultaneously diagonalized [transform $\mathbf{g}$ to $\mathscr{M}_{I}$ using $\operatorname{SL}(3, R)$ and then use an element of $\mathrm{SO}(3, R)$ to diagonalize the resulting $\dot{\mathbf{g}}]$ so it is sufficient to consider only diagonal solution curves, provided of course that the matter potential is $\operatorname{SL}(3, \mathbf{R})$ invariant as in the perfect fluid case. Electromagnetic fields may break the symmetry and excite nontrivial automorphism degrees of freedom.

In the Type II case, $\mathbf{k}_{3}$ vanishes. We might consider replacing this generator by $\mathbf{e}^{1}{ }_{2}-\mathbf{e}^{2}{ }_{1}$, leading to a basis $\left\{\boldsymbol{\kappa}_{a}\right\}=\left\{\mathbf{k}_{1}, \mathbf{k}_{2}, \mathbf{e}^{1}{ }_{2}-\mathbf{e}^{2}{ }_{1}\right\}$ of a special automorphism subgroup whose orbits are transversal to $\mathscr{M}_{D}$. $\left(\mathscr{G}_{33}\right.$ then coincides with the canonical Type IX expression while the Class A formulas for $\mathscr{G}_{11}$ and $\mathscr{G}_{22}$ are evaluated at the canonical Type II structure constant tensor components.) However only the adjoint degrees of freedom associated with the first two elements of the basis can be eliminated using the two linearly independent momentum constraints and one is stuck with three degrees of freedom in the end corresponding to the fact that the momentum reduction technique only determines a shift which transforms the curve $\mathrm{g}(t)$ to $\mathscr{M}_{\mathrm{S}(3)}$ using the action of the adjoint group. Yet it appears that the rotational degree of freedom is just as inessential as the three rotational degrees of freedom in the previous case, at least when $U_{M}$ is invariant under this rotation. All we need to do is check whether or not arbitrary initial data tangent to $\mathscr{M}_{S(3)}$ can be obtained from initial data tangent to $\mathscr{M}_{D}$ under the action of the automorphism group. But this is just the two-dimensional analogue of the Type I discussion considering the submatrices obtained by eliminating the third rows and third columns, since the corresponding $\operatorname{SL}(2, R)$ subgroup of $\operatorname{GL}(3, R)$ is a subgroup of the Type II automorphism group. Therefore when the matter potential is invariant under this $\operatorname{SL}(2, R)$ subgroup (i.e. involves only $g$ and $g_{33}$ ), we can set the rotational degree of freedom to zero.

Similarly $\mathbf{k}_{3}^{0}$ vanishes in the Type $\mathrm{V}$ case and we might consider replacing it with the same rotation generator: $\left\{\boldsymbol{\kappa}_{a}\right\}=\left\{\mathbf{e}^{3}{ }_{1}, \mathbf{e}^{3}{ }_{2}, \mathbf{e}^{1}{ }_{2}-\mathbf{e}^{2}{ }_{1}\right\}$. The only modification of the above scheme is that $Q_{3}$ vanishes and $p_{3}$ drops out of the third momentum constraint, so that $p_{1}, p_{2}$, and $p_{+}$are determined by the constraints. In other words the momentum reduction seems to lead not to $\mathscr{M}_{D}$ but the threedimensional submanifold of $\mathscr{M}_{S(3)}$ on which $\beta^{+}=0$ in the corresponding parametrization. The same would be true if we used any traceless linear combination of $\mathbf{e}^{1}{ }_{2}$ and $\mathbf{e}^{2}{ }_{1}$ for $\boldsymbol{\kappa}_{3}$. Unfortunately $\beta^{+}$does not correspond to an automorphism and so remains in the Hamiltonian and thus one is left with three degrees of freedom after the energy reduction. However, exactly as in the Type II case the rotational degree of freedom is fictitious as long as the matter potential only involves $g$ and $g_{33}$.

Having set up machinery to study all the Bianchi types, the first question to systematically investigate is to what submanifolds of the phase space corresponding to submanifolds of $\mathscr{M}$ does the dynamical system on the full phase space 
naturally restrict. The obvious submanifolds of $\mathscr{M}$ in connection with this are $\mathscr{M}_{S(a)}$ (the "symmetric cases"), $\mathscr{M}_{D}$ (the diagonal case), $\mathscr{M}_{T(a)}$ (the "Taublike cases") and $\mathscr{M}_{I}$ (the "isotropic case"). The existence of such cases is often accompanied by discrete or continuous spacetime symmetries and depends on the matter source. This has been discussed elsewhere [12].

Some of these additional symmetries are particularly interesting in connection with "minisuperspace linearization instabilities" [16] of the vacuum and electromagnetic spacetimes, namely those generated by spatially homogeneous Killing vector fields (with the exception of Types I and II where $e_{a}=\tilde{e}_{a}$ and $e_{3}=\tilde{e}_{3}$ respectively due to the degeneracy of the adjoint group). For Types $\mathrm{VII}_{0}$ and VIII, $e_{3}$ is an additional spacetime Killing vector field when the metric matrix curve is confined to $\mathscr{M}_{T(3)}$, while in the Type IX case this is true for each Taublike case. In the electromagnetic case, $e_{3}$ generates a symmetry of both the geometry and the $\mathrm{SH}$ electromagnetic field provided that in addition the electric and magnetic fields are both aligned along $e_{3}$ in the Type $\mathrm{VII}_{0}$ and VIII cases, with corresponding statements holding for each of the Type IX Taublike cases. On these submanifolds of the total phase space, the momentum constraint $\mathscr{H}_{3}=\overrightarrow{\mathscr{H}}\left(\mathrm{e}_{3}\right)=0$ and its linearization $D \mathscr{H}_{3}=0$ both vanish identically. (We are fixing our attention on $\mathscr{M}_{T(3)}$.) The second derivative $D^{2} \mathscr{H}_{3}=0$ (which is just Moncrief's nonlinear constraint [16]) then provides the condition on the tangent vectors to the phase space at these submanifolds which makes them tangent to curves in the constraint subspace. In other words the constraint subspace is bifurcating at these submanifolds within the minisuperspace momentum phase space, providing finite-dimensional examples exhibiting all of the features discussed by Fischer and Marsden in the general setting [17]. A similar bifurcation associated with the energy constraint occurs in the vacuum Type I case at the flat space initial data $\pi=0$ when $e_{\perp}=\partial / \partial t$ becomes a SH Killing vector field.

Acknowledgement. The author would like to thank A. H. Taub, M. P. Ryan, Jr. and L. Smarr for stimulating discussion and R. Ruffini for his instrumental encouragement of this work.

\section{References}

1. Warner, F.W.: Foundations of differentiable manifolds and Lie groups. Glenview, Ill.: Scott, Foresman and Co. 1971; Sagle A. A., Walde, R.E.: Introduction to Lie groups and Lie algebras. New York: Academic Press 1973; Herman, R.: Differential geometry and the calculus of variations. New York: Academic Press 1968

2. Fischer, A.E., Marsden, J.E.: J. Math. Phys. 13, 546 (1972)

3. Misner, C.W.: Minisuperspace. In: Magic without magic. Klauder, J.R. (ed.). San Francisco: W.H. Freeman 1972

4. DeWitt, B.S.: Phys. Rev. 160, 1113 (1967)

5. York, J.W., Jr. : Phys. Rev. Letters 28, 1082 (1972): J. Math. Phys. 14, 456 (1973)

6. Fischer, A.E.: The theory of superspace. In: Relativity. Carmeli, M., Fickler, S. I., Witten, L. (eds.). New York: Plenum 1970

7. Estabrook, F.B., Wahlquist,H.D., Behr, C.G.: J. Math. Phys. 9, 497 (1968); Ellis, G.F.R., MacCallum, M. A.H.: Commun. math. Phys. 12, 108 (1969)

8. Bianchi, L.: Lezioni sulla teoria dei gruppi finite di transformazioni, p. 550 (1918); Collected Works of Bianchi, Vol. 9, pp. 17-109: Sugli spazi a tre dimensioni che ammettono un gruppo continuo movimenti 
9. Misner, C.W.: Phys. Rev. 186, 1319 (1969)

10. Misner, C.W., Thorne, K.S., Wheeler, J.A.: Gravitation. San Francisco: W.H. Freeman 1973

11. Smarr, L., York, J.W., Jr.: Kinematical conditions in the construction of a spacetıme. Phys. Rev. D (to appear)

12. Jantzen, R.T.: Spatially homogeneous cosmology: Background and dynamics. In: Relativistic cosmology and Bianchi universes. Ruffini, R. (ed.). San Francisco: W. H. Freeman 1979

13. Whittaker, E.T.: A treatise on analytical dynamics. Cambridge: Cambridge University Press 1965

14. Ryan, M.P., Jr., Shepley, L.C.: Homogeneous relativistic cosmologies. Princeton, NJ : Princeton University Press 1975; Ryan, M.P., Jr.: J. Math. Phys. 15, 812 (1974)

15. Ryan, M.P., Jr.: Ann. Phys. 65, 506 (1971); 68, 541 (1971)

16. Moncrief, V.: J. Math. Phys. 16, 493 (1975); 17, 1893 (1976); Arms, J.: J. Math. Phys. 18, 830 (1977)

17. Fischer, A.E., Marsden, J.E.: Symmetry breaking in general relativity. (to appear)

Communicated by R. Geroch

Received April 28, 1978

\section{Note Added in Proof}

The author has really assumed that the shift vector field is confined to $\operatorname{aut}(G) \oplus \tilde{\mathfrak{g}}=\operatorname{aut}(G) \oplus \mathfrak{g}$. (The equality holds since $\{\tilde{X}-X \mid X \in \mathfrak{g}\} \subset$ aut $(G)$ generates the inner automorphisms.) However, since $\tilde{\mathfrak{g}}$ generates the left translations which have trivial action on left invariant tensor fields, it is only $g$ and those vector fields which generate outer automorphisms that count. Also, elements of $\tilde{\mathfrak{g}}$ are automatically solutions of the minimal distortion shift equation since they are Killing vector fields of each left invariant metric on $G$. 